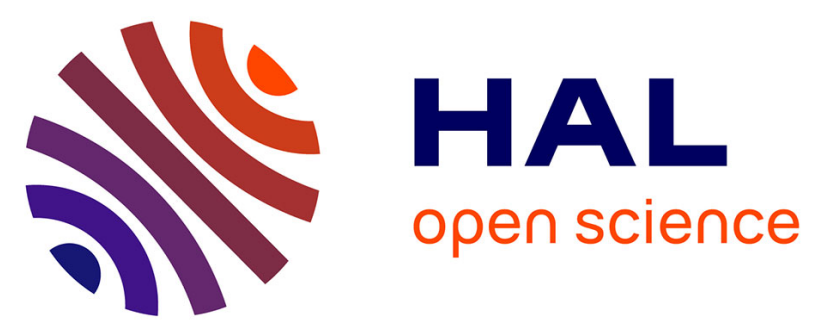

\title{
Estimation of the Seismic Moment Rate from an Incomplete Seismicity Catalog, in the Context of the InSight Mission to Mars
}

Martin Knapmeyer, Brigitte Knapmeyer-endrun, Ana-catalina Plesa, Maren Böse, Taichi Kawamura, John Clinton, Matt Golombek, Sharon Kedar, Simon C Stähler, Jennifer Stevanović, et al.

\section{To cite this version:}

Martin Knapmeyer, Brigitte Knapmeyer-endrun, Ana-catalina Plesa, Maren Böse, Taichi Kawamura, et al.. Estimation of the Seismic Moment Rate from an Incomplete Seismicity Catalog, in the Context of the InSight Mission to Mars. Bulletin of the Seismological Society of America, 2019, 109 (3), pp.1125-1147. 10.1785/0120180258 . insu-02544967

\section{HAL Id: insu-02544967 https://hal-insu.archives-ouvertes.fr/insu-02544967}

Submitted on 26 Aug 2020

HAL is a multi-disciplinary open access archive for the deposit and dissemination of scientific research documents, whether they are published or not. The documents may come from teaching and research institutions in France or abroad, or from public or private research centers.
L'archive ouverte pluridisciplinaire HAL, est destinée au dépôt et à la diffusion de documents scientifiques de niveau recherche, publiés ou non, émanant des établissements d'enseignement et de recherche français ou étrangers, des laboratoires publics ou privés. 
Knapmeyer, M., Knapmeyer-Endrun, B., Plesa, A., Bose, M., Kawamura, T., Clinton, JF., Golombek, MP., Kedar, S., Stahler, S., Stevanovic, J., Perrin, C., Lognonne, P., Teanby, N., \& Weber, R. (2019). Estimation of the Seismic Moment Rate from an Incomplete Seismicity Catalog, in the Context of the InSight Mission to Mars. Bulletin of the Seismological Society of America. https://doi.org/10.1785/0120180258

Peer reviewed version

Link to published version (if available):

$10.1785 / 0120180258$

Link to publication record in Explore Bristol Research

PDF-document

This is the author accepted manuscript (AAM). The final published version (version of record) is available online via GSA at https://pubs.geoscienceworld.org/ssa/bssa/article/570243/estimation-of-the-seismic-moment-ratefrom-an . Please refer to any applicable terms of use of the publisher.

\section{University of Bristol - Explore Bristol Research}

General rights

This document is made available in accordance with publisher policies. Please cite only the published version using the reference above. Full terms of use are available: http://www.bristol.ac.uk/pure/user-guides/explore-bristol-research/ebr-terms/ 
1

3

4

5

6

7

$8 \quad$ J.F. Clinton

$9 \quad$ M.P. Golombek

10

11

12

13

14

15

16

17

18

19

20

M. Knapmeyer

A.-C. Plesa

M. Böse

T. Kawamura

S. Kedar

S. Stähler

J. Stevanović

C. Perrin

P. Lognonné

N.A. Teanby

R.C. Weber

Rutherfordstr. 2
Estimation of the seismic moment rate from an incomplete seismicity catalog, in the context of the InSight mission to Mars

B. Knapmeyer-Endrun

Corresponding Author:

Martin Knapmeyer

DLR Institute for Planetary Research

page 1 of 76, 16352 words 
Seismic Moment Rate Estimation, v 2.0.3, last saved 08/01/2019 10:11:00

$21 \quad 12489$ Berlin

22 martin.knapmeyer@dlr.de

23 phone: 0049-30-67055394

24

page 2 of 76,16352 words 
Seismic Moment Rate Estimation, v 2.0.3, last saved 08/01/2019 10:11:00

We evaluate methods to estimate the global seismic moment rate of a planet from the $k \geq 1$ largest events observed during a limited and possibly short time span, as can be expected e.g. for lander missions to Mars. The feasibility of the approach is demonstrated with a temporary broadband seismometer that was recording in the Mojave Desert, California, for 86 days in 2014, and by application to the Global

31 Centroid Moment Tensor catalog, subsets thereof, and a catalog of stable continental regions seismicity .

32 From the largest event observed at Goldstone alone $\left(M_{W} \approx 7.9\right)$ we estimate the Earth's global moment

33 rate to be $1.03 \times 10^{22} \mathrm{Nm} / \mathrm{yr}$, while an estimation based on the 10 strongest events yields a rate of

$345.79 \times 10^{21} \mathrm{Nm} / \mathrm{yr}$. Summation of 42 years of GCMT solutions result in an average of $7.61 \times$ $3510^{21} \mathrm{Nm} / \mathrm{yr}$. In general, a two years interval of GCMT solutions is sufficient to estimate the Earth's 36 annual moment rate to within a factor 5 or better. A series of numerical experiments with more than 560 37 million synthetic catalogs based on the Tapered Gutenberg-Richter distribution shows that the estima38 tion is rather insensitive against an unknown slope of the distribution, and that bias and variance of the 39 estimator depend on the ratio between moment rate and corner moment of the size frequency distribu40 tion. Moment rates of published Mars models differ by a factor 1000 or more. Tests with simulated cata41 logs show that it will be possible to reject some of these models with data returned by NASA's InSight 42 mission after two years of nominal mission life time. 


\section{Introduction}

The InSight mission (Banerdt et al., 2013) to Mars was launched on May 5, 2018 from Vandenberg Air

47 Force Base, California, and touched down softly in the targeted landing ellipse on Elysium Planitia, Mars,

on November 26, 2018. A combined very broad band and short period seismometer was deployed on

the surface on 19th of December 2018 and is intended to operate until the end of 2020 (Lognonné \&

Pike, 2015, Lognonné et al., submitted).

51 Models for the seismicity of planet Mars usually estimate the long-term average annual seismic mo-

52 ment rate (Table 1), and also the average annual event rate. This holds for estimations based on geologi-

53 cal evidence (Golombek et al., 1992, Golombek, 2002, Taylor et al., 2013) as well as for models based on

54 thermal evolution and cooling of the Martian lithosphere (Phillips, 1991, Knapmeyer et al., 2006, Plesa et

55 al., 2018). All studies are compatible with the conclusion drawn from the non-observation of any unam-

56 biguous seismic event by the Viking missions (Anderson et al., 1977, Goins \& Lazarewicz, 1979), i.e. that

57 Martian seismicity ranges somewhere between that of the Moon and that of Earth. However the range

58 of predicted moment rates is wide and observational constraints of moment rate are therefore desirable

59 to reject models and further our understanding of planetary heat engines. Here we investigate to what

60 extent the InSight mission can be expected to contribute to the rejection of seismicity models. The main

61 obstacles are that the detection threshold of a single station will be relatively high compared to a global

62 network, while the expected life time is relatively short compared to the instrumental record available

63 for Earth. Our approach basically relies on most of the seismic moment being released in a few, large

64 events, and employs Monte Carlo methods to assess how likely any given seismicity model results in the moment rate estimation obtained from the recorded marsquakes.

67 time and station spacing of seismic networks: On the Moon, the first long period seismometer was de- 
Seismic Moment Rate Estimation, v 2.0.3, last saved 08/01/2019 10:11:00

68

ployed in 1969, later augmented by four more stations to form a triangle (Apollo 12 and 14 were relatively close together, compared with Apollo 15 and 16). The network was shut down in late 1977 (Vostreys, 1980). One seismometer (Viking 2) was in operation on Mars from September 1976 to April 1978, i.e. for about 20 months (Toksöz, 1979). The Venera 13 and 14 missions each landed one seismometer on Venus on the $1^{\text {st }}$ of March 1982 (Venera 13) and $3^{\text {rd }}$ of March 1982 (Venera 14), respectively, and with lander life times of 127 and 57 minutes (Siddiqi, 2002). During these times, about 1.5 minutes of seismic waveforms and, additionally, statistical waveform parameters derived on board, were downlinked to Earth (Ksanfomaliti et al., 1982). The Huygens probe contained a suite of accelerometers to monitor its path through the atmosphere of Titan and to record the touchdown shock. Although not intended to conduct a surface seismic experiment, the HASI accelerometer onboard the Huygens probe continued to record data for approximately $30 \mathrm{~min}$ after touchdown (Hathi et al., 2009). A network of three triaxial accelerometers was deployed on comet 67P/Churuyomov-Gerasimenko within the landing gear of Rosetta's lander Philae, resulting in a network aperture of about $2.5 \mathrm{~m}$ (Knapmeyer et al., 2016). From this experiment, about $70 \mathrm{~s}$ of high-frequency data (sampling rates up to $16 \mathrm{kHz}$ ) were returned. With a design life time of two terrestrial years, InSight will significantly surpass these recent experiments.

An instructive comparison to the much better coverage of the Earth arises from the Global Centroid Moment Tensor project (GCMT, Dziewonski et al., 1981, Ekström et al., 2012). At the same time the first seismometers were launched to Mars, the GCMT started producing its catalog of Earthquake source mechanisms, complete to magnitudes of about five and currently based on data from nearly 200 seismic observatories distributed worldwide. The GCMT continuously contributed to the picture of Earth's moment-frequency distribution for the whole 42 years during which there was not even a single seismometer on Mars.

Besides the relatively short period of time covered by extraterrestrial seismicity catalogs so far, spatial coverage and completeness thresholds result in additional obstacles for the estimation of distribu- 
92 tion parameters. Even within the Apollo network on the Moon, the most extensive network outside

93 Earth so far, distance between stations was around $30^{\circ}$, i.e. the network triangle covered only a small

94 fraction of the lunar surface. Limited spatial coverage results in a distance dependent detection thresh-

95 old: small events will be detected only in the vicinity of the network or station, if there is but one. In

96 turn, large, globally detectable events will be overrepresented in the catalog such that the moment-

97 frequency distribution appears to be less steep than it actually is. Also, the estimation of event rates

98 must rely on assumptions about source distribution in distant areas in order to extrapolate the detected

99 local or regional seismicity to the entire planet.

100 The seismic moment of Earthquakes is predominantly released by the few largest events. This sug101 gests that it is possible to obtain a reasonable estimate of the seismic moment rate from evaluating only 102 a small number of large events, or even from the single largest event ever observed. Central questions 103 are how such an estimate can be obtained and how accurate it will be, and also what accuracy is neces104 sary to make a rate estimation a useful constraint to improve our understanding of Mars.

To answer the latter, we consider the moment rate estimates summarized in Table 1. Moment rates given there for Earth and Moon differ by almost exactly ten orders of magnitude, or 4.7 moment magni107 tude units. The individual estimations for Mars cover a range of 2.1 moment magnitude units, or a factor 108 of approximately 1150 in the seismic moment. It is thus desirable to estimate the moment rate with accuracy better than this in order to reject some or several of the published seismicity models.

In the following, we will first describe our proposed approaches for moment rate estimation, and how 111 we generate synthetic seismicity catalogs for numerical testing (section "Methods"). The available cata112 logs are described in section "Data". Since no event catalog is currently available for Mars, we briefly 113 summarize the seismotectonic background information available for Mars. To test if reasonable results 114 can be obtained, we evaluate several seismicity catalogs for the Earth, discuss the applicability to the 115 Moon, and evaluate synthetic catalogs in a generic parameter study and for Mars-specific models (sec- 
Seismic Moment Rate Estimation, v 2.0.3, last saved 08/01/2019 10:11:00

116 tion "Results"). Section "Single Station Completeness Threshold" specifically demonstrates that a single

117 broadband station is sufficient to obtain a meaningful moment rate estimate for an entire planet within

118 a relatively short time.

120 As Kagan \& Schoenberg (2001) point out, a statistical consideration of magnitudes rather than mo-

121 ments is advantageous by transforming multiplicative uncertainties into additive ones. Besides, taking

122 the logarithm of the moment transforms the numerical values in to a more convenient range.

123 Throughout this work, we will frequently convert seismic moments $M$ into equivalent moment magni-

124 tudes $m$ via

$$
m=\frac{2}{3}\left(\log _{10} M-9.1\right)
$$

(after Bormann et al., 2002). Using this formula, we also express moment rates as if the entire mo-

127 ment was released in a single event. All magnitudes used throughout this paper are moment magnitudes

128 following from the above equation, unless indicated otherwise. The coefficient $2 / 3$ implies that a factor

129 of 1000 in the moment $M$ corresponds to a difference of exactly 2 moment magnitude units. We denote

130 seismic moments by uppercase letters and magnitudes by lowercase letters to avoid the excessive index-

131 ing that would result from the conventional lettering, and two indexed quantities $M_{x}$ and $m_{x}$ refer to

132 moments and magnitudes connected by the above equation.

133 The coefficients of eq. 1 originate in a calibration of the seismic moment scale to other magnitude 134 scales and thus carry a certain heritage of Earth's seismological properties, e.g. via the decay of surface 135 waves over distances not available e.g. on Mars. It must be kept in mind that relations to seismogram 
Seismic Moment Rate Estimation, v 2.0.3, last saved 08/01/2019 10:11:00

136

137

138 amplitudes and, moreover, intensities will differ between Earth, Mars, and other bodies. Appropriate magnitude scales for Mars have been developed by Böse et al. (2018).

\section{Seismic Moment Rate Estimation}

Following the seminal works by Gutenberg \& Richter $(1941,1944)$, the size-frequency distribution of earthquakes is often described by what we now call a power law, although Gutenberg \& Richter were aware that the frequency of very large events cannot follow the same law as the frequencies of small and medium ones for physical reasons. Assuming a fault plane as large as the Earth's surface, and with a slip proportional to the square root of the fault area as in the relations given in Knapmeyer et al. (2006), we find a resulting seismic moment of $7 \times 10^{28} \mathrm{Nm}$ (corresponding to $m=13.2$ ) being a hard upper limit for quake size on Earth, as any geologically plausible fault would have a smaller rupture area. The extrapolation of the linear part of the size-frequency distribution of the GCMT predicts that events of this size occur every 10000 to 100000 years: The classical Gutenberg-Richter law, when interpreted naively, predicts that ridiculously large events recur on time scales that are short compared to e.g. the Wilson cycle. Given that there are no traces at all of such events, we take it as an observational fact that this prediction is not true and the size-frequency distribution must bend down significantly somewhere between $5.3 \times 10^{22} \mathrm{Nm}$ (the Tohoku event of March 2011) and $10^{29} \mathrm{Nm}$.

We assume that the moment frequency distribution follows a tapered Pareto distribution (a.k.a. tapered Gutenberg-Richter distribution), which basically is a Gutenberg-Richter law tapered with an exponential function for the largest events (Figure 1).

In this distribution, the cumulative relative number of events with a seismic moment larger than $M$ is given by (Kagan, 2002a, b, and references therein)

$$
\Phi(\mathrm{M})=\left(\frac{M_{t}}{M}\right)^{\beta} \exp \left(\frac{M_{t}-M}{M_{c}}\right) \text { for } M_{t} \leq M<\infty
$$


Seismic Moment Rate Estimation, v 2.0.3, last saved 08/01/2019 10:11:00

158

159

160

161

162

163

164

es one and $\dot{N}(M) \propto \dot{M}_{S} M_{c}^{\beta-1}$, which plots as a straight line in log-log coordinates, such that the ob-

Here $M_{c}$ is the "corner moment" where the exponential taper gains dominance over the classical Gutenberg-Richter power law relation, $M_{t}$ is the threshold moment above which the catalog is complete (not to be confused with the detection threshold $M_{d}$, i.e. the smallest moment for which the catalog contains some, but not all, events), and $\beta$ is a slope parameter. The part of the graph of Figure 1 for $M_{d} \leq M \leq M_{t}$, i.e. the incomplete detection of events between detection threshold $M_{d}$ and completeness threshold $M_{t}$, is not described by eq. 2 as it is at least partially controlled by effects and properties not related to the quake production, like background noise, anelasticity, and instrument sensitivity.

To obtain absolute numbers, $\Phi(M)$ has to be multiplied with a calibration factor following Kagan (2002b)

$$
\alpha_{0}=\frac{\dot{M}_{S} M_{c}^{\beta}(1-\beta)}{M_{t}^{\beta} M_{c} \Gamma(2-\beta) \exp \left(M_{t} / M_{c}\right)}
$$

where $\dot{M}_{S}$ is the moment release rate per unit time of events exceeding $M_{t}$, and $\Gamma(x)$ is the Gamma function with $\sqrt{\pi / 4} \leq \Gamma(2-\beta) \leq 1$ for $0.5 \leq \beta \leq 1$ (e.g. Weisstein, undated). The absolute rate $\dot{N}(M)$ of events with a seismic moment larger than $M$ is thus obtained from combining eqs. 2 and 3

$$
\dot{N}(M)=\alpha_{0} \Phi(M)
$$

In addition to the rate $\dot{M}_{S}$ we introduce the cumulative moment $M_{S}$ such that one event of size $M_{S}$ per unit time would account for the entire moment rate.

\footnotetext{
served event rate allows inferences on $\dot{M}_{S} M_{c}^{\beta-1}$.
} 
For any catalog of observed events, one can estimate $\dot{N}(M)$ via the number $N$ of events listed in the

178 catalog (not taking into account those below $M_{t}$ ), and the time span covered by it.The moment rate can

179 be estimated by summing up all events and dividing by the number of unit time intervals covered by the

180 catalog, as was done with the GCMT in Table 1.

181 The total moment released can be computed by integration over the distribution, but since the moment rate is a factor in $\dot{N}(M)$ we can save the integration and solve $\dot{N}(M)$ for $\dot{M}_{S}$

$$
\dot{M}_{S}=\dot{N}(M) \frac{\Gamma(2-\beta)}{1-\beta} \frac{M^{\beta}}{M_{c}^{\beta-1}} \exp \left(\frac{M}{M_{c}}\right)
$$

Although $M_{t}$ cancelled out, $\dot{M}_{S}$ is still only the moment rate resulting from events exceeding $M_{t}$ by implication via $\dot{N}$. If $M_{t} \ll M_{c}$ and $\beta<1$, the difference to the moment rate resulting from all events is however neglible.

Now we consider only the largest event that ever occurred. By definition, the largest event ever (or LVR, for short, from Largest eVent eve $R$ ) is contained in the catalog only once. Thus, if the catalog covers 189 a time interval of $n$ unit intervals, we identify $M=M_{L V R}$ and obtain $\dot{N}\left(M_{L V R}\right)=1 / n$, and eq. 5 allows estimating the global moment rate from a single event, provided the corner moment $M_{c}$ of the distribu191 tion and its slope parameter, or reasonable estimates, are given.

Using eq. 2 we find the relative frequencies of events exceeding $M_{c}$ by a factor $x$ is

$$
\frac{\Phi\left(x M_{c}\right)}{\Phi\left(M_{c}\right)}=\left(\frac{1}{x}\right)^{\beta} \exp (1-x)
$$

With a convenient (and not entirely unrealistic) value of $\beta=2 / 3$, this results in a drop of frequency magnitude units larger is reduced by $10^{436}$ with respect to the corner moment. Thus the former are 

well.

The largest event ever hence poses a (statistical) lower limit $M_{L V R} \leq M_{c}$ simply by its existence. guess. Inserting this into eq. 5 yields

$$
M_{S} \approx \frac{1}{n} \frac{\Gamma(2-\beta)}{1-\beta} M_{L V R}
$$

and we call this the normalized largest event ever, NLVR for short, estimation of the moment rate.

The middle term approaches infinity for $\beta \rightarrow 1$, but in reality $1 / 2 \leq \beta \leq 2 / 3$ appears to hold, from which $\sqrt{\pi} \leq \Gamma(2-\beta) /(1-\beta) \leq 2.68$ follows.

We define $n$ based on the duration of the observation: if the unit time interval has a duration $\Delta T$, seismicity is recorded between $t_{\text {start }}$ and $t_{\text {end }}$ (where $t_{\text {start }}$ is not necessarily the time of the first event, and $t_{\text {end }}$ is continuously growing in an ongoing experiment), then the normalization factor for the largest event ever which occurred at a time $t_{L V R}$ between start and end is $n=\left(t_{\text {end }}-t_{\text {start }}+1\right) / \Delta T$, and also continuously grows in an ongoing experiment.

With this definition, $1 / n$ and thus the estimated value of $\dot{M}_{S}$, will approach zero for $t_{\text {end }} \rightarrow \infty$. Mathematically, this appears to be a flaw, but in the long run the observer can take the onset of a continuous decay as indication that the largest event ever has exceeded the corner moment of the distribution.

A more sophisticated estimation of $M_{c}$ is given by Kagan \& Schoenberg (2001). As they found that a maximum likelihood estimation of $M_{C}$ is severely biased for small catalogs, they derive an alternate estimator based on expressions for the first and second statistical moment of the tapered Gutenbergcataloged events, the standard deviation $\sigma$ of the $M_{i}$, and the mean moment 


$$
\bar{M}=\frac{1}{k} \sum_{i=1}^{k} M_{i}
$$

they obtain the estimator

$$
\widetilde{M}_{c}=\frac{\sum\left(M_{i}^{2} / k\right)-M_{t}^{2}}{2\left[M_{t}^{2} \beta+(1-\beta) \bar{M}\right]}
$$

from which a bias correction

$$
\widetilde{M}_{\text {bias }}=\frac{(\beta-1)\left[2 M_{t}^{3}+3 M_{t}^{2} \widetilde{M}_{c} \beta+\left(\sigma^{2}+\bar{M}^{2}\right)\left(6 \widetilde{M}_{c}-3 \widetilde{M}_{c} \beta-2 \bar{M}\right)\right]}{4 k\left[M_{t} \beta+(1-\beta) \bar{M}\right]^{2}}
$$

$224 \quad$ has to be subtracted. The difference $\widetilde{M}_{c}-\widetilde{M}_{\text {bias }}$ is then substituted for $M_{c}$ in eq. 5 .

225 The estimator $\widetilde{M}_{c}-\widetilde{M}_{\text {bias }}$ holds for i.i.d. events, i.e. the parameters of the earthquake-generating 226 distribution must be constant in time, and the quakes must not trigger each other. Therefore the catalog 227 has to be declustered before estimating the corner moment. We call the combination of equations 5, 9, 228 and 10 the k-events Kagan-Schoenberg, or $\mathrm{KS}_{\mathrm{k}}$ for short, estimator of the moment rate.

229 The $\mathrm{KS}_{\mathrm{k}}$ estimator requires a catalog containing at least $k$ events and a reasonable estimate about the 230 completeness threshold $M_{t}$ of that catalog to be computable and useful. If only a single event is availa231 ble, one can either equate $M_{t}$ with the size of that event, resulting in $\widetilde{M}_{c}=0$, or assume that $M_{t}$ is 232 smaller than the event, resulting in $\widetilde{M}_{c}$ being dependent only on that assumption but not on the ob233 served event. Moreover, $\sigma$ and hence $\widetilde{M}_{\text {bias }}$ are undefined for a single event. Therefore the $\mathrm{KS}_{\mathrm{k}}$ estima234 tor cannot be used with a single event.

235 The event magnitudes or seismic moments in a real marsquake catalog will carry an uncertainty as 236 discussed by Böse et al. (2018). This uncertainty for individual events is propagated into the uncertainty 237 of NLVR and $\mathrm{KS}_{\mathrm{k}}$ estimations in a Monte Carlo fashion by evaluating a number of modified catalogs, 238 where each event's seismic moment is perturbed according to a normal distribution with the standard 
Seismic Moment Rate Estimation, v 2.0.3, last saved 08/01/2019 10:11:00

239 deviation cataloged for this individual event, and taking care for error propagation through conversion

240 between different types of magnitudes or magnitudes and seismic moments.

\section{Emission Probabilities}

242 Due to the stochastic nature of the tectonic process generating the real quakes, as well as of any cata-

243 log simulation process, simulated catalogs will never match observed quake sequences on an event-by244 event basis even if the model underlying the simulation matches the real process perfectly, and all pa245 rameters are known exactly.

246 Due to the limitations of the data set we expect to obtain for Mars, we do not consider a maximum247 likelihood fit of an assumed type of distribution in order to estimate its parameters feasible.

248 The moment rate estimation described above is intended to be a direct estimation of one of the pa249 rameters of the size-frequency distribution, but is likely to be biased and carry some variance, not the 250 least due to the uncertainty of the seismic moments of observed events. Bias and variance will depend 251 on several factors, such as the parameters of the Tapered Gutenberg-Richter distribution themselves, 252 and the period of time covered by the simulated catalog.

253 We borrow (without intending to over-stress the analogy) from the field of hidden layer Markov 254 chains the term "emission probability" for the probability that a stochastic process produces a statistical 255 diagnostic with the same value as obtained from another, inaccessible process: In our case, a quake cata256 log is a realization of a stochastic process which is transformed into a single-valued diagnostic using one 257 of our estimators. This transformation is a secondary stochastic process of which the resulting diagnostic 258 is a single realization. We aim to estimate the probability that a numerical simulation of seismicity cata259 logs results in the same moment rate estimations as the observed catalog.

260 The catalog simulation we describe in the next section is a random number generator with three pa261 rameters: moment rate, corner moment, and distribution slope. These parameters form a state space, 
Seismic Moment Rate Estimation, v 2.0.3, last saved 08/01/2019 10:11:00

262 which we scan on a search grid with a regular node spacing when moment rate and corner moment are

263 expressed as magnitudes following equation 1. For each node, we produce a large number of synthetic 264 catalogs and evaluate the NLVR and $\mathrm{KS}_{\mathrm{k}}$ estimators for all these catalogs, resulting in a distribution of 265 estimations. Comparing these distributions with the respective estimations of the real catalog, we can 266 determine the probability that a certain combination of state parameters makes the catalog simulation 267 emit a moment rate estimation within the uncertainty limits of the moment rate estimation derived 268 from the real catalog.

This finally yields a map of emission probabilities for the scanned part of the state space, which can 270 then be compared with predicted parameters e.g. from Knapmeyer et al. (2006), or with values for Earth 271 and Moon.

272 Examples will be shown below in sections dealing with seismicity catalogs for Earth and Moon, and 273 synthetic catalogs for Mars. These are computed on a search grid with node spacing of 0.1 moment 274 magnitudes in both moment rate and corner moment dimensions. For each grid node, we evaluate 1000 275 catalogs, resulting in 3.1 million catalogs for the depicted grids. We evaluate for each node the probabil276 ity that a given state emits a value within 0.2 magnitude units (corresponding to a factor 2 in seismic 277 moment) from the respective target values as given in figure titles (the GCMT comes without uncertainty 278 ranges for the scalar moment, therefore we use a fixed interval width here). Computation time on a 279 desktop computer fits into a lunch break and thus leaves room for refining the search grid or evaluating 280 more catalogs per grid node.

\section{Simulation of Seismicity Catalogs}

Equation 2 only predicts the relative frequencies of events. In order to reproduce the seismicity observed during a time interval of duration $\Delta t$, it needs to be normalized in such a way that also the abso-

284 lute number of events $N(M)$ and the observed cumulative moment $M_{\text {cum }}=\dot{M} \Delta t$ released by all events 
Seismic Moment Rate Estimation, v 2.0.3, last saved 08/01/2019 10:11:00

285 in the catalog are reproduced. Following Kagan (2002b), the long term average $\alpha_{0}$ is computed by evalu286 ating eq. 3.

We adjust the - in our context entirely fictitious - completeness threshold $M_{t}$ such that we obtain catalogs of approximately 10000 events.

The number $N_{\Delta t}$ of events in an actual time interval, e.g. a given calendar year, follows a Poisson distribution describing the probability that the value of $N_{\Delta t}$ is $n$ (e.g. Zhuang et al., 2012)

$$
P\left\{N_{\Delta t}=n\right\}=\frac{\lambda}{n !} e^{-\lambda}
$$

where $\lambda$ is the rate parameter of the distribution.

For a rate parameter exceeding about 10, the Poisson distribution can be approximated by a normal

distribution with mean $\bar{x}=\lambda$ and standard deviation $\sigma=\sqrt{\lambda}$. For time intervals of the order of years and a sufficiently low detection threshold, one can expect hundreds or thousands of quakes, such that this condition is met. We thus draw the number of events per time interval from a normal distribution 297 with $\lambda=\alpha_{0}$.

The distribution of earthquakes in time (when disregarding aftershocks) also follows a Poisson distri299 bution, as was first shown for California by Gardner \& Knopoff (1974). With the number $n$ of events oc300 curring during interval $\Delta \mathrm{T}$ already being given by eqs. 3 and 11 , is it thus sufficient to distribute the 301 source times uniformly over the time interval (see Zhuang \& Touati, 2015, algorithm 10).

302 Finally, for each source time generated thus, a seismic moment is drawn from the tapered Pareto dis303 tribution, following the method described by Vere-Jones et al. (2001), which we reproduce here for self304 containedness. We first generate a first set $U_{1}$ of $n$ uniformly distributed random numbers from the in305 terval $] 0,1[$. From these we compute 
Seismic Moment Rate Estimation, v 2.0.3, last saved 08/01/2019 10:11:00

$$
y=U_{1}^{-1 / \beta}
$$

In addition, we employ the inverse method to produce $n$ exponentially distributed random numbers inverse of the exponential distribution

$$
X=-\frac{1}{\lambda} \log \left(1-U_{2}\right)
$$

to obtain a set $X$ of exponentially distributed random numbers, where

$$
\lambda=\frac{M+M_{t}}{M_{c}}
$$

is the rate parameter of the exponential distribution $f(\tau)=\lambda \exp (-\lambda \tau)$. Finally,

$$
K=\left(M+M_{t}\right) * \min (y, 1+X)-M_{t}
$$

is a tapered Pareto distributed seismic moment value following the distribution given in eq. 2 .

It should be noted that assuming a Poisson process is equivalent to the assumption that the individual events are entirely independent of each other, i.e. the process has no memory of the past. This assumption does not apply to foreshock/aftershock sequences, therefore a comparison of real catalogs with synthetic ones generated with the above procedure requires the declustering of the real catalog. Moreover, this kind of catalog simulation might be unsuitable for sources with a periodic driving force, such as deep moonquakes (DMQ).

It has been argued that the slope parameter $\beta$ is actually not a free parameter but a universal con323 stant with a value $\beta=1 / 2$ (initially apparently by Vere-Jones, 1976, recently e.g. by Kagan, 2014). Anal324 yses of different tectonic settings (Kagan, 1997), Flinn-Engdahl regions (Kagan, 1999) and rectangular 325 regions defined by parallels and meridians (Godano \& Pingue, 2000) support that $\beta$ is a global constant 
Seismic Moment Rate Estimation, v 2.0.3, last saved 08/01/2019 10:11:00

326 for quakes on Earth (except mid-oceanic ridges), however with a value $0.6 \leq \beta \leq 2 / 3$. Kagan (2014)

327 argues that the deviation from the prediction is a combination of several observational biases.

328 Thermal moonquakes appear to follow a distribution where most of the moment is released by the 329 small events (implying $\beta>1$, Cooper \& Kovach, 1975), and it is questionable that deep moonquakes, 330 with a stress source that reverts direction on a monthly scale, fall under the same universal relation as 331 subduction zone events resulting from a uniformly progressing slab. So although we follow Knapmeyer et 332 al. (2006) that $\beta=0.625$ is a good initial choice for Mars, lacking a better one for now, we are aware 333 that this needs to be established experimentally, and it must not be taken for granted.

\section{Data}

The following subsections describe three seismicity catalogs for the Earth and one catalog for the Moon that were used as test cases for moment rate estimation. In the absence of a Mars seismicity catalog we give a brief summary of Martian seismotectonic properties that make us conclude that earthquake statistics methods based on terrestrial circumstances are applicable to Mars as well.

\section{GCMT catalog}

342 We downloaded the complete catalog of the Global Centroid Moment Tensor project (GCMT, 343 Dziewonski et al., 1981, Ekström et al., 2012) from IRIS (see "Data and Resources"). This catalog covers 344 the 15456 days from 05. January 1976 (date of the first event in the GCMT) to 17. May 2018, or 42.32 345 years of 365.25 days, and contains 49178 events with seismic moments between $3.49 \times 10^{15} \mathrm{Nm}$ and $3465.32 \times 10^{22} \mathrm{Nm}$. The completeness threshold for the catalog as a whole is $M_{t} \approx 2 \times 10^{17} \mathrm{Nm}$, but de- 
Seismic Moment Rate Estimation, v 2.0.3, last saved 08/01/2019 10:11:00

347

348

350

351

\section{Stable Continental Regions catalog} recent years. events) released $90 \%$ of $\sum M_{0}$. $6.6 \times 10^{20} \mathrm{Nm}\left(M_{w}=7.8\right)$.

\section{Temporary Installation at Goldstone, CA}

creases with time; especially the year 1976 is significantly less complete, while $M_{t}$ is lower for the more

The cataloged events released a cumulative seismic moment of $\sum M_{0}=3.23 \times 10^{23} \mathrm{Nm}$. The ten largest events in this catalog released $47.5 \%$ of $\sum M_{0}$, and the 615 largest events (i.e. $1.25 \%$ of all

The Stable Continental Regions catalog of Schulte and Mooney (2005, see also "Data and Resources" represents a compilation for several sources of historically witnessed events and of events for which instrumental recordings exist, and covers the entire Earth for the time from 495 AD to 2003. Schulte and Mooney have estimated seismic moments for most of the 1373 events in the catalog. Cataloged moments range from $5.3 \times 10^{15} \mathrm{Nm}$ to $7.5 \times 10^{23} \mathrm{Nm}$, the latter however is assigned to a reservoirassociated event in India in September 2000 and is most likely erroneous. We exclude this and another reservoir-associated event for which a moment of $>10^{23} \mathrm{Nm}$ is given from our analysis, leaving as the largest listed event an earthquake that occurred in July 1819, also in India, with a seismic moment of

For each event, the catalog indicates if instrumental records, historical desriptions, or both are available. For events after 1976, the catalog is informed by the GCMT.

A single Nanometrics Trillium Compact seismometer (120 s eigenperiod) was installed temporarily during summer 2014, in a dry lake bed in the Mojave desert, approximately $60 \mathrm{~km}$ NNE of Barstow, California, and approximately $1 \mathrm{~km}$ southwest of the $70 \mathrm{~m}$ "Mars" antenna dish of NASA's Deep Space Network (Lorenz et al., 2015). The purpose of this experiment was to gather seismic and microbarometric 
Seismic Moment Rate Estimation, v 2.0.3, last saved 08/01/2019 10:11:00

recordings of dust devils, to support the interpretation of dust devil recordings expected from Mars. The experiment was not intended to record earthquakes, but these are of course hard to avoid.

The surface of the lake bed is composed of fine mud that grades into denser sediment overlying a rock with increasing seismic velocities with depth, which is likely analogous to the InSight landing site (see Lorenz et al., 2015, Golombek et al., 2017, and Knapmeyer-Endrun et al., 2017). The seismometer was shallowly buried and accompanied by a microbarometer and an anemometer on the surface. The seismic sampling frequency was $100 \mathrm{~Hz}$, but we resampled to $2 \mathrm{~Hz}$ to simulate the continuous data stream that will be available from InSight (Lognonné et al., submitted). The seismometer operated from 05. June 2014 to 29. Aug. 2014. Several downtime periods add up to 18 days ( $24 \mathrm{~h}$ periods, not to be confused with the $24.6 \mathrm{~h}$ of the martian rotation period) of lost data, such that a total of 68 days of seismic recordings remains.

During this experiment, the USGS National Earthquake Information Center (NEIC) catalog lists 304 global events with magnitudes of 5 and larger.

We convert the 7 different types of magnitudes found in the NEIC catalog $\left(M_{S}, m_{b}, M_{L}\right.$, and several versions of $\mathrm{M}_{\mathrm{W}}$ ) into seismic moments to quantify source strength, using relations from Wason et al. (2012) , and from Bormann et al. (2002).

By visual inspection, we categorize all events according to their signal quality with categories given in Table 2. We expect that magnitudes or seismic moments could be determined from InSight data for events comparable to categories $A$ through $E$, and sufficiently precise locations to reduce the distance effect in magnitude uncertainty to below 0.3 magnitude units for categories $A, B$, and $E$ (Böse et al., 2018). 
Seismic Moment Rate Estimation, v 2.0.3, last saved 08/01/2019 10:11:00

391

\section{Moonquakes, deep and shallow}

Seismic observation of Apollo has detected more than 13000 seismic events (Nakamura, 2003; see

"Data and Resources"), encompassing endogenous moonquakes, meteorite impacts, and artificial sources.

The most frequent type of events is the deep moonquakes (DMQ) at depths between $700 \mathrm{~km}$ and $1100 \mathrm{~km}$ (Nakamura, 2005). They occur in clusters which are activated and deactivated in cycles corresponding to the orbital periods of the Moon-Earth-Sun system. Time lags between individual events of individual clusters are also not random (Bulow et al., 2007, Weber et al., 2010, and references therein). Kawamura et al. (2017) show that stress drops associated with DMQ are of the order of magnitude of the tidal stresses in their source regions. The DMQ are thus not only triggered but also caused by tidal stresses, i.e. the orbital motions in the Moon-Earth-Sun system can be considered as a part of the DMQ source mechanism. Since orbital motion is described by differential equations that allow evaluation forward and backward in time, the DMQ mechanism has a memory of the past and therefore cannot be i.i.d. Poissonian. The situation appears to be different for the (poorly understood) shallow moonquakes, which we discuss in section "Moon: High Frequency Teleseisms".

Therefore, we focus on shallow moonquakes in the following. The source mechanism of shallow moonquakes is not yet clear but they are likely to be caused by thermal contraction of the Moon (e.g. Binder \& Lange, 1980), i.e. the same mechanism expected for marsquakes (Phillips, 1991, Knapmeyer et al., 2006, Plesa et al., 2018). If this is correct, shallow moonquakes can be regarded as lunar analogs of marsquakes.

\section{Martian Seismotectonics}

The seismic data available from Mars so far (up to late November 2018) contains only one candidate marsquake, which was recorded by the Viking 2 seismometer (Anderson et al., 1977), but could not iden- 
Seismic Moment Rate Estimation, v 2.0.3, last saved 08/01/2019 10:11:00

414 tified as such unambiguously. At the time of writing, we are thus not in the possession of a seismic data 415 set that allows for statistical evaluation. In this section we describe in more detail what is known about 416 the seismotectonics of Mars, in order to support that out statistical approach is meaningful for Mars as 417 well as for Earth.

418 After the successfuldeployment on December 19, 2018, broad band and short period seismic data 419 from the InSight lander is expected to be received from January 2019 onwards. Routine evaluation of 420 events, encompassing the identification of seismic phases, epicenter location based on multi-orbit sur421 face waves and polarization analysis (Böse et al., 2017, Panning et al., 2015), will be conducted by a team 422 at the Swiss Seismological Service located at ETH Zürich (Clinton et al., 2018), the Marsquake Service 423 (MQS). Mars-specific magnitude scales were developed (Böse et al., 2018) and will be used by the MQS 424 to quantify source strength on Mars. The event catalog produced by the MQS will also discriminate be425 tween different source types (tectonic, impacts, landslides, and atmospheric effects) based on the expe426 rience with Apollo data and physical differences in the source mechanism (see Daubar et al., 2018, con427 cerning impacts, and Clinton et al., 2018, concerning the MQS).

Mars is a one-plate planet which shows no unambiguous indications of subduction zones or midocean ridges, neither in topography nor in free air gravity anomalies or lithospheric magnetic field (e.g. 430 Zuber et al., 2000, Hirt et al., 2012, Morschhauser et al., 2014). Nevertheless, it must have witnessed 431 much seismic activity in the past, as its surface is crisscrossed with complex patterns of normal and 432 thrust faulting. Knapmeyer et al. (2006) mapped more than 8500 normal and thrust faults with a cumula433 tive length of about $680000 \mathrm{~km}$, updated later on to approx. 14800 faults with a cumulative length of $434940000 \mathrm{~km}$ (Knapmeyer et al., 2008). The shortest of these has a length of approx. 600 meters, while the 435 longest graben that dissects the Tharsis bulge is almost $1450 \mathrm{~km}$ long. The Knapmeyer et al. (2006) fault 436 catalog appears to be complete for faults longer than $80 \mathrm{~km}$. The overall size-frequency distribution of 437 Martian faults can be described by a power law with exponent well in the range found for fault popula- 
Seismic Moment Rate Estimation, v 2.0.3, last saved 08/01/2019 10:11:00

438 tions on Earth (see Knapmeyer et al., 2006, and references therein). If one assumes that all fault seg439 ments are equally likely to break, and that large marsquakes require long breaking segments, then the 440 length-frequency distribution of Martian faults is in favor for a power-law-like distribution of Marsquake 441 sizes.

442 Taking the age of the youngest surface crossed by a fault as indicator of its age, no age preference for 443 shorter or longer faults is found, i.e. the size frequency distribution appears to be independent of time. It 444 must be noted, however, that $75 \%$ or more of the total fault length was produced more than 3.5 Ga ago. 445 The youngest faults are estimated to have an age of approx. $300 \mathrm{Ma}$.

$446 \quad$ Fault populations on Mars show features like fault linkage or relay ramps well known from Earth (see 447 Schulz et al., 2010, and references therein, for a general treatment). The mechanisms of fault growth are 448 considered to be the same on Earth and Mars (see Wyrick et al., 2018, for a recent analog study), alt449 hough faults on Mars accumulate less displacement per length than faults on Earth. This can however be 450 attributed to properties that all scale with gravity (Schulz et al., 2006).

451 Stresses resulting from differential cooling of the planetary interior are widely accepted as the main 452 source of Martian seismicity (Phillips, 1991, Knapmeyer et al., 2006). Plesa et al. (2018) also investigated 453 stresses resulting from mantle drag due to convection and found that these might under certain circum454 stances contribute significantly to the moment rate, and especially modify the regional distribution of 455 effective stresses. Knapmeyer et al. (2006) defined end-member and "typical" models with "strong", 456 "weak" and "medium" moment rates of $4.78 \times 10^{18} \mathrm{Nm} / \mathrm{yr}, 3.42 \times 10^{16} \mathrm{Nm} / \mathrm{yr}$, and $5.99 \times$ $45710^{16} \mathrm{Nm} / \mathrm{yr}$, respectively. Due to different corner moments, their models StrongFew, StrongMany, 458 WeakFew, WeakMany, and Medium produce $m=4$ events with recurrence rates between 13 hours and 4596.7 years. Based on these and other estimations of martian seismcity, Panning et al. (2017) expect about 460100 events per year that are detectable at distances beyond $60^{\circ}$. 
Seismic Moment Rate Estimation, v 2.0.3, last saved 08/01/2019 10:11:00

While the Moon's seismic event rate is dominated by the deep moonquakes that are tied to Earth 462 tides, Phillips (1991) found that although tidal loading by the sun produces high strain rates, the associ463 ated strains are small, and concluded that tidal loading of Mars is unimportant from the seismicity point 464 of view. We expect that Mars, unlike the Moon, has no significant periodic seismicity.

Mars is well known for its giant volcanos. These have been active through much of the Martian histo466 ry. Although some of the youngest volcanic surfaces might be only a few million years old(Neukum et al., 467 2004), no actual volcanic activity has ever been reported. The wristwatch pattern of faults around the Alba Patera volcano actually shows that its magma chamber retreated (Cailleau et al., 2003). Volcanic 469 contributions to seismic activity are thus considered unlikely.

$470 \quad$ Fresh impacts have been found on Mars in images taken repeatedly from the same area (Malin et al., 471 2006), and might provide semi-controlled seismic sources when the location of an impact detected by a 472 seismometer can be identified in imagery. Quantitative analysis shows that the recurrence intervals of 473 globally detectable impacts is between one year and 10 years (Teanby \& Wookey, 2011), where the 474 longer intervals are expected to be more likely due to expected noise conditions. Contrary to an intuition 475 one might have based on the cratered surface of especially the southern hemisphere of Mars, impacts 476 are also not expected to dominate the seismic record.

\section{Results}

To assess the reliability of moment rate estimations we conduct numerical tests where we compare estimated rates with the GCMT catalog of earthquakes from the time interval 1976 to 2018 (section 
Seismic Moment Rate Estimation, v 2.0.3, last saved 08/01/2019 10:11:00

483 logs"). Finally, we generate synthetic catalogs for several models of Martian seismicity and investigate if 484 and how we can distinguish between them (section "Mars-specific synthetic catalogs").

485 Earth

Since the GCMT, as the most comprehensive and homogenous long-term catalog of moment tensor 488 solutions, relies on a global network of about 200 stations, we first need to establish that a single station 489 can provide data representative for Earth's global moment rate. The question here is what the global 490 completeness threshold for a single (broadband) seismometer is, and how long it takes until the largest 491 recorded event is above this completeness threshold.

492 We then compare how the output of different estimators evolves in time, i.e. re-evaluate all estima493 tors for each new event in the catalog throughout the time covered. Finally, we compare estimations for 494 different tectonic settings (subduction zones, mid-ocean ridges, and stable continental regions) by subdi495 viding the GCMT catalog according to Flinn-Engdahl-Zones and by evaluation of an additional catalog 496 dedicated to stable continental regions (as an analog for the single plate regime on Mars) which also 497 contains events predating the instrumental recording of earthquakes.

498 Since we will likely not be able to determine reliably the slope parameter of the martian seismicity 499 distribution, we use a fixed value of $\beta=2 / 3$ throughout all these tests.

\section{$500 \quad$ Single Station Completeness Threshold}

501

We use as single station the temporary installation at Goldstone described in section "Temporary In503 stallation at Goldstone, CA". To find its completeness threshold, we compare the size-frequency (survivor) distribution of events detected at Goldstone with the size-frequency distribution of the GCMT 
(Figure 2). Distributions from short recordings are less smooth than that of the whole GCMT data base, and also differ between independent time windows of the same duration. In addition, the magnitude-tomoment conversion is based on empirical relations between uncertain quantities and may thus provide an uncertain moment value only, thus adding to the roughness of the curve. We therefore do not attempt to pin down the completeness threshold very precisely, but only estimate that it is somewhere between $10^{18} \mathrm{Nm}$ and $10^{19} \mathrm{Nm}$ for events of grade $\mathrm{A}$ alone, and possibly lower when taking $\mathrm{A}$ and $\mathrm{B}$, or

$511 \mathrm{~A}, \mathrm{~B}$, and $\mathrm{E}$ together. The GCMT lists 1273 events exceeding $10^{19} \mathrm{Nm}$, and 5891 events exceeding $51210^{18} \mathrm{Nm}$, resulting in recurrence times of 12.2 and 2.7 days, respectively.

513 The strongest events in the grade $A, A \cup B$, and $A \cup B \cup E$ subsets are the same, so it is sufficient to 514 use one of them fora moment rate estimation that relies on the strongest events. From the grade $A$ events alone, we obtain a NLVR moment rate estimate of $1.03 \times 10^{22} \mathrm{Nm} / \mathrm{yr}\left(m_{S}=8.6\right)$, while the $516 \quad \mathrm{KS}_{10}$ estimator returns $5.79 \times 10^{21} \mathrm{Nm} / \mathrm{yr}\left(m_{S}=8.4\right)$.

For comparison, we subdivide the GCMT catalog into 180 non-overlapping time windows, each of which has the same 86 days duration as the Goldstone experiment. Using the NLVR estimator we obtain a moment rate equivalent to magnitude $8.28 \pm 0.42$ from these time windows, while the $\mathrm{KS}_{10}$ estimator results in an equivalent magnitude of $8.25 \pm 0.24$

521 Compared with the $7.61 \times 10^{21} \mathrm{Nm} / \mathrm{yr}\left(m_{S}=8.52\right)$ obtained from the complete GCMT catalog, 522 and comparing with Figure 3 and the $\mathrm{KS}_{10}$ emission probabilities in Figure 4, we conclude that the Gold523 stone experiment suffices to estimate the Earth's global seismic moment rate with acceptable accuracy.

524 One can thus expect that a seismometer installation comparable to the one at Goldstone produces a 525 globally representative "largest event ever" time series three months or less after deployment, meaning 526 that all comparable installations worldwide would produce the same such time series. For any longer recording time it is thus justified to evaluate the GCMT catalog instead of a single station catalog. 
Seismic Moment Rate Estimation, v 2.0.3, last saved 08/01/2019 10:11:00

528

529

530

531

532

533

534

535

\section{Moment Rate Estimations: Entire Earth}

We compare the evolution in time of several different moment rate estimators. For this exercise we use only events from 1977 onwards since the GCMT completeness threshold for 1976 is significantly higher than for later years. We expect that the $N L V R$ and especially the $K S_{k}$ estimators have a certain burn-in time: one cannot evaluate $K S_{k}$ before having cataloged $k$ events. Moreover, if the catalog contains events below the threshold for global detectability, the first listed events will not be representative for the global moment rate. By excluding 1976 from consideration we avoid mixing the burn-in time of the GCMT project with that of the rate estimation.

As a baseline we consider the summation of all events contained in the catalog between a time $T_{\text {start }}$ and up to a time $t$, and divide by the number of years (assuming 365.25 days per year) elapsed between $T_{\text {start }}$ and $t$ to obtain an annual rate. The resulting time series $\dot{M}_{s, b a s e}(t)$ is thus based on all data available at time $t$.

We compare this baseline with estimations based on the single strongest event $\left(\dot{M}_{S, N L V R}(t)\right.$, based on the $N L V R$ estimator) and on the $2,5,10$, and 50 strongest events $\left(\dot{M}_{s, K S k}(t)\right.$, based on the $K S_{k}$ estimator). The latter are computed only after a sufficient number of events is available, thus the respective time series start somewhat later than $\dot{M}_{s, b a s e}(t)$ and $\dot{M}_{s, N L V R}(t)$. All time series are compiled in Figure 3.

A common feature of all time series is that they decay like $1 / t$ or shallower as long as no large new contribution to the moment release occurs. This is of course most pronounced in $\dot{M}_{S, N L V R}(t)$, which can have a new peak only if an event occurs that is larger than any event before. But the effect is also clear in the $\dot{M}_{s, K S k}(t)$ time series, where smaller peaks indicate that new events entered the top- $k$ list. Since new events can enter this list at any position, the value of the $K S_{k}$ estimator will change more often with increasing $k$, hence the time resolution of $\dot{M}_{s, K S k}(t)$ increases with $k$. 


$$
\exp \left(\left|\log \left(\dot{M}_{s, e s t}(t)\right)-\log \left(\dot{M}_{s, b a s e}(t)\right)\right|\right)
$$

to evaluate the deviation between an estimator and the baseline value, as it is sufficient to know that one of the two values has to multiplied by some factor to obtain the other one.

554 All estimators except $K S_{2}$, which sometimes overestimates by three orders of magnitude, follow the 555 full-catalog baseline closely. Even the single-event $N L V R$ estimation never deviates by more than a fac556 tor of nine, as does $K S_{5}$. Both $K S_{10}$ and $K S_{50}$ are always within a factor 2.5 or less from the baseline, 557 most of the time the factor is smaller than 2. When subdividing the GCMT into 21 consecutive, non558 overlapping intervals of 2 years, the KS10 value is always within a factor 5 of the 42-years average, and 559 within a factor 2 in half of all cases. All estimators tend to underestimate. We however do not think that 560 the evaluation of a single catalog is sufficient to declare this a rule.

\section{Moment Rate Estimations: Specific Tectonic Settings}

562 Earth's seismicity is mainly due to plate tectonics, and by far most of its seismicity (approximately 75

$563 \%$ of all events) occurs at active plate boundaries. Subduction zones alone account for approximately 60 $564 \%$ of all earthquakes. Mars, on the other hand, is a one-plate planet. It is thus desirable to investigate if 565 reasonable moment rate estimations can be made for selected, either Earth specific or Mars specific 566 tectonic settings rather than for the entire Earth.

567 Based on the Flinn-Engdahl regionalization scheme of seismic regions (Young et al., 1995), we extract 568 events belonging to mid-ocean ridges and subduction zones from the GCMT according to the region IDs 569 listed in the appendix 0. Since the F-E-Regions define regions on the Earth's surface but not in its interior, 570 we further subdivide the events classified as "subduction zone" into two depth layers, above and below $571100 \mathrm{~km}$, in order to clearly separate subducting slabs from crustal events. The number of events sorted 572 into each of the four resulting groups of events is given in Table 3. 
Seismic Moment Rate Estimation, v 2.0.3, last saved 08/01/2019 10:11:00

573 As reported earlier (e.g. Bird \& Kagan, 2004), the distribution for mid-ocean ridge events appears to 574 be significantly steeper than for the other groups (Figure 5Fehler! Verweisquelle konnte nicht gefunden

575 werden.), the slopes of which appear to be essentially identical with that obtained for the complete 576 catalog. Especially a test using mid-ocean ridge events is thus a scenario different from the other groups, 577 or the GCMT taken as a whole.

578 For each group we evaluate (a) the sum of all events divided by the number of years covered by the 579 catalog (42.3 years), (b) the NLVR estimator, and (c) the $K S_{10}$ estimator, thus obtaining three values for 580 each group. The resulting moment rates, expressed both in $\mathrm{Nm} / \mathrm{yr}$ and as moment magnitude of a sin581 gle event releasing the same amount of seismic moment, are also summarized in Table 3.

582 In most cases, both the $N L V R$ and the $K S_{10}$ estimator underestimate the value obtained by summing 583 up all events of a subset. The ratios between these estimators and the complete summation are well in 584 the range found for the entire Earth. This holds for all four subsets.

585 Thus the results obtained for the GCMT are encouraging, but we note that an evaluation of a station 586 installed e.g. in early 2004 would look different, since its event catalog would report two very strong 587 events (Banda Aceh and Tohoku) within only 6 years.

588 Neither subduction zones nor mid ocean ridges exist on Mars. We thus use the Earthquake Catalog 589 for Stable Continental Regions of Schulte \& Mooney (2005, see also "Data and Resources") as a better 590 analog of martian seismicity.

591 We subdivide the catalog into two groups: the first contains events for which no instrumental record 592 is available, while the second contains events that were interpreted solely based on instrumental records 593 (a third group of events, where historical information and instrumental data is interpreted together, was 594 excluded from our analysis). The goal is to compare moment rate estimations resulting from the histori595 cal events with independent estimations for the instrumental epoch. 
We do not consider the 21 events listed for the time between 495 AD and 1500 AD (only three of which occurred before 1000 AD). This may look like a somewhat arbitrary modification of the catalog, but we think it is justified since an extension of the covered period by 1000 years biases the duration of observation in a more severe way than the omission of 21 events with moment magnitudes below 6.1 biases the released seismic moment. We also delete two reservoir-associated events that occurred in India in 2000, since the catalog assigns unlikely seismic moments beyond $10^{23} \mathrm{Nm}$ (corresponding to $M_{W}>9$ ) to both of them, which are also in clear contradiction with the listed magnitude values (around 5). We thus obtain 226 events from 1500 AD to 1949 and 879 events from 1950 to 2003. Their size604 frequency distributions are shown in Figure 5. Here the group of historical events shows a much lower 605 slope than all other distributions shown. This is a property expected for incomplete catalogs, as this historical events list certainly is. The slope of the distribution of the instrumentally evaluated events is very 607 similar to that of the GCMT, but this is not surprising since many of the corresponding catalog entries are 608 based on the GCMT.

609 The resulting moment rate estimates are consistent within each of the two groups (see Table 3), but 610 the values obtained for the events from the instrumental epoch are also consistently higher than those 611 for the historical events, by factors between 1.7 and 4.1, with the NLVR estimator producing the largest 612 deviation. Although these deviations are still in the range of variability seen for the different estimators 613 in Figure 3, they exhibit a certain trend. If expressed as magnitudes, we find that the differences be614 tween the individual estimates is no larger than the uncertainty attributed to the individual magnitude 615 estimations as listed in the catalog, which is typically 1 magnitude unit, and never below 0.4 magnitude 616 units.

In summary we find that the evaluation of the GCMT is encouraging, although it was purely accidental that its largest events occurred late. The deviations obtained with the Stable Continental Regions catalog might be attributed to the very circumstantial nature and general uncertainty of historic observations, 
Seismic Moment Rate Estimation, v 2.0.3, last saved 08/01/2019 10:11:00

620 but might also point to a systematic shortcoming of our approach. To further investigate bias and vari621 ance of our estimators, we conduct an extensive series of tests with synthetic catalogs.

622 Moon: High Frequency Teleseisms

623 For the shallow moonquakes, we use here the seismic moments estimated by Oberst (1987). In total, 62428 shallow moonquakes were detected by the Apollo network. We evaluated the annual seismic mo625 ment release of the following subsets: 1) All shallow moonquake events; 2) Events during 1972-1973; 3) 626 Events during 1974-1975; 4) Events during 1976-1977; 5) Events during 1973-1974; 6) Events during 627 1975-1976. We tested if the seismic moment release evaluated from two-years subsets differ or agrees 628 with that obtained from the complete observation period. We also tested how fast the obtained seismic 629 moment converges to the reference value obtained with the full dataset. We reevaluate the seismic 630 moment release every time a new shallow moonquake is detected and studied the evolution of obtained 631 seismic moment rates like with the GCMT. The results are summarized in Figure $6 . \mathrm{KS}_{10}$ Emission proba632 bilities for the moment rate resulting from all shallow moonquakes together are shown in Figure 7. The 633 maximum emission probability coincides with the rate obtained from all events. In the case of the Moon, 634 the scattering of obtained seismic moment rate is rather small and the difference is about $50 \%$ in maxi635 mum. The small deviation is likely to be due to the smaller range of magnitude (from $4.7 \times 10^{12} \mathrm{Nm}$ to

$6361.6 \times 10^{15} \mathrm{Nm}$ ) and the small number of events for shallow moonquakes. Likewise, the seismic moment 637 rate converged relatively quickly after 4-5 events. Unlike on the Earth, the possible source of excitation is 638 limited and result in smaller variety of events. This may be the case on Mars as well, allowing to obtain a 639 representative seismic moment rate rapidly.

\section{Generic Synthetic Catalogs}

641 We set up a logarithmically equidistant grid of $M_{S}$ and $M_{c}$ values via an equidistant grid of equivalent 642 magnitudes. We vary $m_{S}$ from 3 to 10 in steps of 0.1 magnitude units. The equivalent magnitude of the 
Seismic Moment Rate Estimation, v 2.0.3, last saved 08/01/2019 10:11:00

643 corner moment is varied relative to $m_{S}$ from $m_{S}-4$ to $m_{S}+4$, also in steps of 0.1 magnitude units. This 644 results in a $81 \times 71=5751$ elements matrix of combinations of moment rates and corner moments.

645 The resulting event rates, computed for an assumed completeness threshold magnitude $m_{t}=2$ 646 range from $10^{-216}$ to $10^{9}$ events per year (some parameter combinations result in even smaller rates 647 that were not numerically resolvable, these cases were excluded from the simulation process). Neither 648 of these extremal values is suitable to produce meaningful catalogs. We therefore adjust the complete649 ness threshold individually for each combination of moment rate and corner moment in order to obtain 650 catalogs of uniform sizes for all cases. A simple iterative search scheme is used to solve eq. 3 for $M_{t}$ nu651 merically for this purpose. In analogy to the planned life time of the InSight mission, we generate cata652 logs covering 2 terrestrial years (or 730 terrestrial days), each containing approximately 10000 events (in 653 a few cases, where this requires $m_{t}>m_{c}$, we force $m_{t}<m_{c}$ and obtain catalogs with about $10^{5}$ 654 events).

655 The result of any moment rate estimation will depend on the individual realization of the catalog 656 generator, depending on what the largest cataloged event actually is. To analyze the resulting variability 657 of the estimated moment rate, we thus generate a total of 1410 catalogs (a number chosen with respect 658 to the overall computation time) for each of the 5751 parameter combinations, resulting in approximate659 ly eight million usable catalogs. The complete simulation can be run overnight on a desktop PC.

For each catalog, we evaluate the NLVR and $\mathrm{KS}_{10}$ estimators and record the resulting bias between 661 the moment rate's equivalent magnitude $m_{s}$ used during catalog generation and the estimated value's 662 equivalent magnitude $m_{e s t}$, defined as

$$
\mathrm{m}_{\text {bias }}=\mathrm{m}_{S}-\mathrm{m}_{\text {est }}
$$


Seismic Moment Rate Estimation, v 2.0.3, last saved 08/01/2019 10:11:00

664

665

666

667

668

669

670

671

672

673

674

675

676

677

678

679

680

681

682

683

684

685

i.e. the bias is positive for underestimation. From the biases obtained for catalogs resulting from the same value of the ratio $M_{S} / M_{c}$ (or from the same difference $m_{S}-m_{c}$ ) we construct distribution densities, median values and the boundaries of $95 \%$ quantiles centered around the median, i.e. intervals which contain $95 \%$ of all bias values.

\section{Estimation with correct slope}

In a first test, we use the same slope $\beta \in\{0.4,0.5,0.6,0.625,2 / 3\}$ during catalog generation as well as during the evaluation. We generate independent sets of approximately 8 million catalogs for the evaluation using either the NVLR estimator or the $K S_{10}$ estimator. Since we already established that the $K S_{k}$ estimator gets better with increasing $k$, we now use a fixed value of $k=10$ which is realistic for Mars and expected to perform well in the light of the evaluation of the GCMT.

If the corner moment is known (which can be expected only in synthetic cases) a two-dimensional histogram relating the bias between true and estimated moment rate to the difference $m_{S}-m_{C}$ can be constructed (left panels in Figure 8 (NLVR) and Figure $\left.9\left(K S_{10}\right)\right)$. Individual moment rate estimates may vary strongly between individual catalogs, by as much as three or more units of equivalent magnitude.

The ratio $M_{S} / M_{C}$ controls systematic variations of distribution median and width, and NLVR and $K S_{10}$ show different behavior: While the NLVR estimator shows the greater variance for $m_{S}-m_{C}<0$, the variance of the $K S_{10}$ estimator is larger for $m_{S}-m_{C}>0$. Both estimators are near unbiased if $m_{S}=m_{C}$

If the correct value of $m_{S}-m_{c}$ is unknown, the 2D distribution densities depicted in the left panels of Figure 8 and Figure 9 reduces to the marginal densities depicted in the right panels. These show that, for $\beta=2 / 3$, the probability of overestimating the moment rate is approximately $10 \%$ for the NLVR estimator, and $41 \%$ for the $K S_{10}$ estimator. The probability that the estimated value is within \pm 1 magnitude 
Seismic Moment Rate Estimation, v 2.0.3, last saved 08/01/2019 10:11:00

686

687

688

689

690

691

692

693

units of the true value is $49 \%$ for the NLVR estimator, and $72 \%$ for the $K S_{10}$ estimator for the given range of possible $m_{S}-m_{c}$ differences.

These percentages depend on the value of the slope parameter $\beta$ and increase as $\beta$ increases. As Figure 10 (NLVR) and Figure $11\left(K S_{10}\right)$ show, the distribution densities get narrower and more symmetric with increasing $\beta$ : The probability that the estimated moment rate is within \pm 1 magnitude units from the true value increases as the distribution narrows, and the probability of an overestimation increases as the distribution become more symmetric about the zero bias.

\section{Estimation with incorrect slope}

As mentioned in the introduction, estimating the slope parameter from a single station catalog is likely biased towards small slopes, since only a fraction of the smaller events will be observed. Since both of our moment rate estimators make use of the slope, we must ask how sensitive the outcome is to an incorrectly guessed slope.

We again generate catalogs with a true slope of $\beta \in\{0.4,0.5,0.6,0.625,2 / 3\}$, but use the same set of values used for the estimation from our synthetic catalogs. We thus obtain a matrix of true and assumed slopes and evaluate the fraction of moment rate estimates that falls within \pm 1 or \pm 2 magnitudes from the true value, and also how likely we overestimate the actual moment rate. As before, we evaluate approximately 8 million catalogs for each combination of true and assumed slope parameter.

In Figure 12 we show the resulting percentages, i.e. the width of distribution densities as shown in Figure 10 and Figure 11, for both the NLVR (top) and $K S_{10}$ (bottom) estimator. Although the width parameters depend on both the true slope and the slope assumed by the estimator, the influence of the latter on the number of cases where the estimated moment rate is within \pm 1 or \pm 2 magnitudes from the true value is much smaller than that of the true slope. It is thus sufficient if the assumed slope is approximately correct within a relatively wide range. This holds for both estimators.

page 33 of 76,16352 words 
Seismic Moment Rate Estimation, v 2.0.3, last saved 08/01/2019 10:11:00

A notable difference is the fraction of cases where the actual moment rate is overestimated: For the

NLV estimator, this fraction depends mainly on the assumed slope and not as much on the true slope,

711 whereas it is about equally sensitive on both values for the $K S_{10}$ estimator.

\section{Mars-specific synthetic catalogs}

713 The tests described so far indicate that variance and bias of both the NLVR and the $\mathrm{KS}_{\mathrm{k}}$ estimator are

714 such that we cannot expect to solve the question of the Martian moment rate unambiguously, by invert-

715 ing some estimated value into a best fitting rate. The emission probability approach using a search grid

716 of moment rates and corner moments is more promising. Here we investigate in how far it is possible to

717 distinguish hypothetical states by these emitted rate estimates, and in how far longer experiment life

718 times support this distinction.

719 We consider the five end-member seismicity models of Knapmeyer et al., (2006), which mark some 720 extreme corners of their parameter space. These models were named WeakFew, WeakMany, Medium, 721 StrongFew, and StrongMany according to their relative moment rates (weak, medium or strong) and 722 event rates (few, medium, or many). Since Knapmeyer et al. (2006) use the truncated rather than the 723 tapered Gutenberg-Richter distribution, we have to convert their maximum seismic moment $M_{\text {Max }}$ into 724 corner moments $M_{C}$ in order to keep the moment rate unchanged when switching to the tapered distri725 bution, using

$$
M_{C}=M_{M a x}\left[\frac{\beta}{\Gamma(2-\beta)}\right]^{1 /(1-\beta)}
$$

727 after Kagan (2002b). The conversion factor amounts to approximately 0.416 for $\beta=2 / 3$, or 0.391 for $728 \beta=0.625$ as used in the five models. Moment rates and corner moments of the models are summarized 729 in Table 4. It is worth noting that $m_{S}-m_{C}$, which proved crucial for variance and bias of the estimators 
Seismic Moment Rate Estimation, v 2.0.3, last saved 08/01/2019 10:11:00

730 in the previous tests, is negative for the StrongFew, Medium, and WeakFew models (but close to zero for

731 Medium), but positive for the StrongMany and WeakMany models (rightmost column of Table 4).

732 To assess the effect of experiment life time, we consider catalogs covering $1,2,4,8,16$, and 32

733 (earth) years. The exponential increase of durations was chosen in order to compensate for the power

734 law decay of event probabilities with magnitude. Considering even longer times appears to be unneces-

735 sary, since no lander with such a life time is known, and since we hope that improved follow-up experi-

736 ments will make the entire discussion obsolete at some time.

737 We generate $10^{6}$ catalogs, with approximately 10000 events per catalog, for each duration, and thus 738 obtain six different probability density functions for estimated moment rates for each of the five models.

739 We repeat this for both the NLVR and the $\mathrm{KS}_{10}$ estimator and compare the resulting probabilities graph-

740 ically (Figure 13 and Figure 14). Independent realizations are used for the two estimators, but given the 741 smooth PDFs that we obtain, we are convinced that this does not affect our results.

742 For both estimators, doubling the experiment life time moves the maximum of the histograms (i.e.

743 the mode) in Figure 13 and Figure 14 by a constant amount towards larger values (meaning a multiplica744 tion by a constant factor on the seismic moment scale). This shows that the choice of an exponential 745 series of life times was appropriate to investigate the long term behavior.

746 The NLVR and $\mathrm{KS}_{10}$ estimators however show a significant difference concerning the width (i.e. stand747 ard deviation) of the distributions: While the NLVR distributions become narrower in most cases (not 748 very much in the case of the WeakFew model, left hand side of Figure 15), the $\mathrm{KS}_{10}$ standard deviation 749 decreases not as much, or even grows (in the case of the WeakMany and StrongMany models, right 750 hand side of Figure 15).

751 The changes in mode and standard deviation of NLVR work together in such a way that probabilities 752 left of the maximum decrease quickly with time, while probabilities right of the maximum increase only 
Seismic Moment Rate Estimation, v 2.0.3, last saved 08/01/2019 10:11:00

753 slowly with time. This makes the evolution of the curves look like a caterpillar pulling its rear end to754 wards the head before moving its head forward.

755 The $\mathrm{KS}_{10}$ estimator behaves differently: here the whole curve moves towards the right essentially un756 changed like the shell on the back of a crawling snail, with the notable exception of the StrongMany 757 model which behaves like a caterpillar pushing its head forward towards the right with its tail fixed 758 (Figure 14).

759 The two estimators also behave differently when it comes to the rejection of models.

After two years of registration, NLVR moment rate estimates between $2 \times 10^{16} \mathrm{Nm}$ and $1.3 \times$ 761 $10^{18} \mathrm{Nm}$ (equivalent magnitudes $m_{S}$ between 4.8 and 6) would be rather inconclusive, since all five 762 models have significant probabilities to produce estimates in this range. An estimated rate of less than $3.9 \times 10^{16} \mathrm{Nm}\left(m_{S}=5\right)$ would exclude the StrongMany model. A NLVR estimation below $1 \times 10^{16} \mathrm{Nm}$ $764\left(m_{S}=4.6\right)$ would additionally exclude the Medium and StrongFew models. A NLVR result larger than $7651.6 \times 10^{17} \mathrm{Nm}\left(m_{S}=5.4\right)$ obtained after two years, on the other hand, would exclude the WeakFew, WeakMany and StrongMany models.

With the $\mathrm{KS}_{10}$ estimator, the StrongMany model could be excluded if the rate estimate is below 768 $8.9 \times 10^{17} \mathrm{Nm}\left(m_{S}=5.9\right) . \mathrm{KS}_{10}$ estimates below $5.6 \times 10^{16} \mathrm{Nm}$ or above $1.6 \times 10^{17} \mathrm{Nm}\left(m_{S}\right.$ between 5.1 and 5.4) are crucial to distinguish between the WeakMany, Medium, and StrongFew models. More such thresholds could be identified.

771 As a sketch for the application to InSight data, we simulate a single two years catalog for the Knap772 meyer et al. (2006) Medium scenario with parameters as given in Table 4, and evaluate the $\mathrm{KS}_{10}$ estima773 tor. This results in an estimated moment rate of $2.72 \times 10^{17} \mathrm{Nm} / \mathrm{yr}$. The emission probability map 774 favors moment rates close to the one used for catalog generation, and clearly allows the rejection of a 775 moment rate as low as the Moon's (Figure 16). Based on the simulated catalog, a moment rate as high as 
Seismic Moment Rate Estimation, v 2.0.3, last saved 08/01/2019 10:11:00

776 the Earth's could only be accepted when assuming that marsquakes larger than the largest known earth777 quakes are possible.

778 The following observations are crucial here: Firstly, in principle, it is possible to reject at least some of 779 the Knapmeyer et al. (2006) models using the NLVR estimator alone. Second, the moment rate thresh780 olds to exclude models evolve with time, i.e. with the time span covered by the catalog. And third, 781 thresholds excluding individual models differ between the NLVR and $\mathrm{KS}_{10}$ estimators. Also, the use of the $782 \quad \mathrm{KS}_{10}$ estimator was a decision made here to keep the amount of tests to run in a reasonable range. If 783 more events become available, there is however no reason not to use them for a $K S_{k>10}$ estimation. It is 784 thus meaningful to use different estimators in parallel, and to also track the evolution of the estimators 785 with time.

\section{Discussion}

Our tests with terrestrial data demonstrate that the Earth's moment rate can be estimated from a single, short-lived broadband station, even if it is a temporary installation rather than being placed in a 789 state of the art observatory shelter (the Lorenz et al., (2015) installation at Goldstone serves as an exam790 ple). The list of the largest $k$ events becomes representative within a few weeks. This minimum time 791 frame of course depends on the actual moment and event rates and is likely longer on Mars. It will be a 792 crucial exercise to determine from the observations what the duration of this "burn in" time on Mars is. 793 Besides the usual methods to determine the completeness threshold of catalogs, a comparison of the 794 actual Mars catalog with modeled detection probabilities might be useful. For the Earth, the moment 795 rate can be estimated to within a factor 2.5 or better after a burn in of less than 2 weeks from the 10 796 largest events (Figure 3). 
Seismic Moment Rate Estimation, v 2.0.3, last saved 08/01/2019 10:11:00

A subdivision of Earth's seismicity into specific tectonic settings (subduction zones, subducting slabs alone, mid-ocean ridges, stable continental regions) still allows estimating the moment rate of these regions within the uncertainty of the seismic moments of the individual events used. For the stable continental settings we however obtain larger deviations, indicating that either the approach is less reliable than the whole-GCMT results suggest, or that the estimators show a significant variance.

To assess more generic properties of our estimators, we conduct a series of Monte Carlo experiments, simulating seismicity catalogs for a variety of parameters, and compare the estimated moment rates with the known values used for the simulation.

The estimation of the uncertainty of the bias estimate critically depends on the assumed range of 806 $M_{S} / M_{C}$, while knowledge of the slope $\beta$ is of minor importance, thus allowing to use a generic value like $807 \beta=2 / 3$. To our best knowledge, there is no self-consistent limitation of $M_{S} / M_{C}$ in the origins of the 808 tapered Gutenberg-Richter distribution. Maximum likelihood estimation of $M_{C}$ often produces a lower 809 limit only (Bird \& Kagan, 2004), and any estimator will suffer from the fact that the necessary large 810 events are also the most rare ones. Thus giving a range of $M_{S} / M_{C}$ is hardly possible in advance and will 811 remain challenging in the course of the experiment, as far as the InSight mission to Mars is concerned. 812 We limited the difference of equivalent magnitudes to $\left|m_{S}-m_{C}\right|<4$, motivated by the range $813-2.4<m_{S}-m_{C}<1.7$ found with the end-member models of Knapmeyer et al. (2006), and adding 814 some margin.

815 A physical argument creating an upper limit for the corner moment arises from geological considera816 tions on the largest possible event, as discussed in section "Seismic Moment Rate Estimation" for the 817 Earth, assuming an event with a fault plane as large as the planet's surface.

818 The estimation for the seismic moment upper bound used there scales with $R^{3}$, thus the correspond819 ing estimation for Mars is about $15 \%$ of that obtained for Earth, or $10^{28} \mathrm{Nm}$ (or $M_{W}=12.6$ ). The crater- 
Seismic Moment Rate Estimation, v 2.0.3, last saved 08/01/2019 10:11:00

820 ing record and derived surface ages for Mars show that no event involving the entire Martian surface 821 occurred during the recent 3.8 billion years or so (taking the systematic age difference between Hesperi822 an northern lowlands and Noachian southern highlands as a reference for the creation of the dichotomy, 823 whatever caused it), the longest faults of Knapmeyer et al., (2006) actually suffice for $M_{W} \approx 9$ if the en824 tire fault breaks in a single event. We thus predict that the corner moment of the Martian quake statistic 825 is such that events of $M_{W}=12.6$ do practically not occur in the sense of the discussion in section 826 "Seismic Moment Rate Estimation", i.e. that the corner moment is at least one magnitude below $82710^{28} \mathrm{Nm}$ and does not exceed $10^{25} \mathrm{Nm}$ (this is however a weak prediction given that the Knapmeyer et 828 al. (2006) models hardly exceed $10^{20} \mathrm{Nm}$ for good reasons).

829 The InSight mission will have to deal with a physically reasonable subset of all possible parameters.

830 Testable predictions that can be challenged individually by InSight data have been made in the literature, 831 and a determination of the moment rate sufficiently accurate to reject some models would be a signifi832 cant achievement.

833 Our simulations reveal that both the NLVR and $\mathrm{KS}_{\mathrm{k}}$ estimations show considerable biases and vari834 ances. Nevertheless the NLVR estimator, as the most simple one, will allow constraining the actual mo835 ment rate of Mars when comparing its output with simulated distributions of given seismicity models. 836 Depending on experiment life time and the chosen estimators, threshold exists that clearly discriminate 837 between the Knapmeyer et al. (2006) models. The most promising approach for the interpretation of 838 moment rate estimates appears to be to scan the moment-rate-corner-moment state space like in Figure 8394 , Figure 7, and Figure 16, and determine the probability that a certain state emits the moment rate es840 timated from observed events.

841 Our approach relies on several assumptions:

842 - Marsquakes are i.i.d. events resulting from a Poisson process. 
- Their size-frequency distribution can be described by the Tapered Gutenberg-Richter distribution.

- The slope of the distribution is such that the moment rate is governed by the strongest, ra-

It might not be possible to test all these assumptions with InSight data alone. Establishing the validity of the Tapered Gutenberg-Richter distribution, for example, will likely require much longer observation times and more than one station - even the decade-long catalogs available for Earth do not yet provide a clear picture of the distribution of the strongest earthquakes. All these assumptions, however, can be tested in principle. We intend to evaluate several additional diagnostics based on event location and distribution of events in time to assess the validity of our assumptions.

\section{Conclusion}

859 Statistics is best when it is based on a large data set. The boundary conditions of interplanetary mis860 sions however make it challenging to obtain quake catalogs comparable to the GCMT. We nevertheless 861 have to make the most of whatever data we obtain.

862 With equations (2) and (3), the tapered Gutenberg-Richter distribution of quakes is described by

863 slope $\beta$, corner moment $M_{C}$, moment rate $\dot{M}_{S}$, and an observer-dependent completeness threshold 864 moment $M_{t}$. While we frequently find statements in the literature that thousands of quakes are neces- 
Seismic Moment Rate Estimation, v 2.0.3, last saved 08/01/2019 10:11:00

865

866

868 earthquakes. Secondly, we use synthetic catalogs generated from the Tapered Gutenberg-Richter distri-

872 bution and represented by a total of more than 560 million catalogs worth of almost 2.5 billion years of 873 simulated seismicity.

874 We find that the proposed estimators will allow rejecting at least some of the published seismicity

875 models by their predicted moment rates. The uncertainty of the estimates depends on $\dot{M}_{S} / M_{C}$, but is 876 sufficiently small to discriminate several published seismicity models even after only two years mission 877 life time on Mars.

879 The Apollo seismic event catalog can be found at http://www880 udc.ig.utexas.edu/external/yosio/PSE/catsrepts/

881 The Global Centroid Moment Tensor was retrieved from IRIS, 882 https://ds.iris.edu/ds/products/momenttensor/ on 17. May 2018.

883 Waveform data from the Goldstone experiment was obtained from Lorenz et al. (2015), we kindly re884 fer to their Data and Resources section. 
Seismic Moment Rate Estimation, v 2.0.3, last saved 08/01/2019 10:11:00

885

886

887

888

889

890

891

892

893

894

The Stable Continental Regions catalog of Schulte \& Mooney (2005) was retrieved from the USGS at https://earthquake.usgs.gov/data/scr_catalog.php, last accessed 12. June 2018.

The coordinates of Flinn-Engdahl region boundaries was retrieved from USGS, at ftp://hazards.cr.usgs.gov/web/hazdev-geoserve-ws/FE/, last accessed 15.05.2018

\section{Acknowledgements}

We wish to thank Associate Editor Nicholas van der Elst, Peter Bird (UCLA) and two anonymous reviewers for their comments which helped to clarify the manuscript. We are also grateful to Betty Schiefelbein of the BSSA editorial office for generous support concerning formatting issues.

This work benefitted from discussions within the InSight SEIS science team, and especially R. WidmerSchnidrig of Black Forest Observatory and D. Giardini of ETH Zürich.

A portion of the work was supported by the InSight Project at the Jet Propulsion Laboratory, California Institute of Technology, under a contract with the National Aeronautics and Space Administration.

R.C.Weber was supported by the NASA InSight project, contract NNH10ZDA0070.

N. Teanby was supported by the UK Space Agency.

This is InSight Contribution Number 77.

Anderson, D.L., Miller, W.F., Latham, G.V., Nakamura, Y., Toksöz, M.N., Dainty, A.M., Duennebier, F.K., Lazarewicz, A.R., Kovach, R.L., Knight, T.C.D. (1977). Seismology on Mars, J. Geophys. Res., vol. 82, No. 28, 4524-4546

Banerdt, W.B., Smrekar, S., Hurst, K., Lognonné, P., Spohn, T., Asmar, S., Banfield, D., Boschi, L., Christensen, U., Dehant, V., Folkner, W., Giardini, D., Goetz, W., Golombek, M., Grott, M., Hudson, T., Johnson, C., Kargl, G., Kobayashi, N., Maki, J., Mimoun, D., Mocquet, A., Morgan, P., Panning, M., Pike, W.T., 
Seismic Moment Rate Estimation, v 2.0.3, last saved 08/01/2019 10:11:00

907

908

909

910

911

912

913

914

915

916

917

918

919

920

921

922

923

924

925

926

927

928

929

930

931

932

933

934

935

936

937

938

939

940

941

942

943

944

945

946

947

948

Tromp, J., van Zoest, T., Weber, R., Wieczorek, M. and the InSight Team, (2013). InSight: A discovery mission to explore the interior of Mars, 44th., Lunar Planet. Sci. Conf., abstract 1915

Binder, A.B., Lange, M.A. (1980). On the thermal history, thermal state, and related tectonism of a Moon of fission origin; J. Geophys. Res., vol. 85, B6, 3194-3208

Bird, P.; Kagan, Y.Y. (2004) Plate-Tectonic analysis of shallow seismicity: apparent boundary width, beta, corner magnitude, coupled lithosphere thickness, and coupling in seven tectonic settings, Bull. Seis. Soc. Am., vol. 94, No. 6, 2380-2399

Bormann, P., Baumbach, M., Bock, G., Grosser, H., Choy, G.L., Boatwright, J. (2002). Chapter 3: Seismic Sources and Source Parameters, In: Bormann, P. (Ed.)(2002) IASPEI New Manual of Seismological Observatory Practice, GeoForschungszentrum Potsdam, vol. 1

Böse, M., Clinton, J., Ceylan, S., Euchner, F., van Driel, M., Khan, A., Giardini, D., Lognonné, P., Banerdt, W.B., (2017) A probabilistic framework for single-station location of seismicity on Earth and Mars, Phys. Earth Planet. Inter., vol. 262, 48-65, doi:10.1016/j.pepi.2016.11.003

Böse, M., Giardini, D., Stähler, S., Ceylan, S., Clinton, J.F., van Driel, M., Khan, A., Euchner, F., Lognonné, P., Banerdt, W.B. (2018), Magntiude scales for Marsquakes, Bull. Seis. Soc. Am., vol. 108, No. 5A, 27642777, doi: 10.1785/0120180037,

Bulow, R.C., Johnson, C.L., Bills, B.G., Shearer, P.M. (2007) Temporal and spatial properties of some deep moonquake clusters, J. Geophys. Res., vol. 112, doi:10.1029/2006JE002847

Cailleau, B., Walter, T.R., Janle, P., Hauber, E. (2003) Modeling volcaninc deformation in a regional stress field: Implications for the formation of graben structures on Alba Patera, Mars, J. Geophys. Res., vol. 108 (E12), 5141, doi:10.1029/2003JE002135

Clinton, J., Giardini, D., Böse, M., Ceylan, S., van Driel, M., Euchner, F., Garcia R.F. Kedar, S., Khan, A., Stähler, S.C., Banerdt, B., Lognonné, P., Beucler, E., Daubar, I., Drilleau, M., Golombek, M., Kawamura, T., Knapmeyer, M., Knapmeyer-Endrun, B., Minoun, D., Mocquet, A., Panning, M., Perrin, C., Teanby, N.A. (2018) The Marsquake Sercive: Securing Daily Analysis of SEIS Data and Building the Martian Seismicity Catalogue for InSight, Space Sci. Rev., vol. 214, doi:10.1007/s11214-018-0567-5

Cooper, M.R., Kovach, R.L. (1975). Energy, frequency, and distance of moonquakes at the Apollo 17 site, Proc. Lunar Sci. Conf. 6th, pp. 2863-2879

Daubar, I., Lognonné, P., Teanby, N.A., Miljkovic, K., Stevanivić, J., Vaubailln, J., Kenda, B., Kawamura, T., Clinton, J., Lucas, A., Drilleau, M., Yana, C., Collins, G.S., Banfield, D., Golombek, M., Kedar, S., Schmerr, N., Garcia, R., Rodriguez, S., Gudkova, T., May, S., Banks, M., Maki, J., Sansom, E., Karakostas, F., Panning, M., Fuji, N., Wookey, J., van Driel, M., Lemmon, M., Ansan, V., Böse, M., Stähler, S., Kanamori, H., Richradson, J., Smrekar, S., Banerdt, W.B., (2018), Impact-Seismic Investigations of the InSight Mission, Space Sci. Rev., vol. 214, doi: 10.1007/s11214-018-0562-x

Dziewonski, A. M., Chou, T.-A., Woodhouse, J.H (1981), Determination of earthquake source parameters from waveform data for studies of global and regional seismicity, J. Geophys. Res., vol. 86, 2825-2852, doi:10.1029/JB086iB04p02825

Ekström, G., Nettles, M., Dziewonski, A.M. (2012), The global CMT project 2004-2010: Centroid-moment tensors for 13,017 earthquakes, Phys. Earth Planet. Inter., vol. 200-201, 1-9, doi:10.1016/j.pepi.2012.04.002

Gardner, J.K.; Knopoff, L. (1974). Is the Sequence of Earthquakes in Southern California, with Aftershocks removed, Poissonian?, Bull. Seism. Soc. Am., vol. 64, No. 5, 1363-1367

page 43 of 76,16352 words 
Seismic Moment Rate Estimation, v 2.0.3, last saved 08/01/2019 10:11:00

949

950

951

952

953

954

955

956

957

958

959

960

961

962

963

964

965

966

967

968

969

970

971

972

973

974

975

976

977

978

979

980

981

982

983

984

985

986

987

988

Godano, C., Pingue, F. (2000). Is the seismic moment-frequency relation universal?, Geophys. J. Int., vol. 142, 193-198

Golombek, M.P., Banerdt, W.B., Tanaka, K.L, Tralli, D.M. (1992). A prediction of mars seismicity from surface faulting, Science, vol. 258, No. 5084, 979-981

Golombek, M.P. (2002). A revision of Mars seismicity from surface faulting, Lunar Planet. Sci. Conf., XXXIII, Abstract 1244

Golombek, M., Kipp, D., Warner, N., Daubar, I.J., Fergason, R., Kirk, R.L., Beyer , R., Huertas, A., Piqueux, S., Putzig, N.E., Campbell, B.A., Morgan, G.A., Charalambous, C., Pike, W.T., Gwinner, K., Calef, F., Kass, D., Mischna, M., Ashley, J., Bloom, C., Wigton, N., Hare, T., Schwartz, C., Gengl, H., Redmond, L., Trautmann, M., Sweeney, J., Grima, C., Smith, I.B., Sklyanskiy, E., Lisano, M., Benardini, J., Smrekar, S., Lognonné, P., Banerdt, W.B. (2017) Selection of the InSight Landing Site, Space Sci. Rev., vol. 211, 5-95, DOI 10.1007/s11214-016-0321-9

Gutenberg, B., Richter, C.F. (1941). Seismicity of the Earth, in: Seismicity of the Earth, Geological Society of America Special papers No. 34, Geological Society of America, New York, 1-126

Gutenberg, B., Richter, C.F. (1944). Frequency of earthquakes in California, Bull. Seis. Soc. Am., vol. 34, No. $4,185-188$

Hathi, B., Ball, A.J., Colombatti, G., Ferri, F., Leese, M.R., Towner, M.C., Withers, P., Fulchigioni, M., Zarnecki, J.C. (2009). Huygens HASI servo accelerometer: A review and lessons learned, Planetary and Space Science, vol. 57, 1321-1333, doi:10.1016/j.pss.2009.06.023

Hirt, C., Claessens, S.J., Kuhn, M., Featherstone, W.E. (2012) Kilometer-resolution gravity field of Mars: MGM2011, Planet. Sp. Sci., vol. 67, 147-154, doi:10.1016/j.pss.2012.02.006

Kagan, Y.Y, (1997). Seismic moment-frequency relation for shallow earthquakes: regional comparison, J. Geophys. Res., vol. 102, B2, 2835-2852

Kagan, Y.Y. (1999). Universality of the Seismic Moment-frequency Relation, Pure Appl. Geophys., vol. $155,537-573$

Kagan, Y.Y. (2002a). Seismic Moment Distribution revisited: I. Statistical results. Geophys. J. Int., vol. 148, 520-541

Kagan, Y.Y. (2002b). Seismic moment distribution revisited: II. Moment conservation principle, Geophys. J. Int. vol. 149, 731-754

Kagan, Y.Y. (2014). Earthquakes. Models, Statistics, Testable Forecasts, American Geophysical Union, Wiley, 283 pages

Kagan, Y.Y., Schoenberg, F. (2001). Estimation of the upper cutoff parameter for the tapered pareto distribution, J. Appl. Probab., vol. 38A, 158-175

Kawamura, T., Lognonné, P., Nishikawa, Y., Tanaka, S. (2017), Evaluation of deep moonquake source parameters: Implication for fault characteristics and thermal state, J. Geophys. Res. Planets, vol. 122, 1487-1504, doi:10.1002/2016JE005147

Knapmeyer, M., Schneider, S., Misun, M., Wählisch, M., Hauber, E. (2008) An extended global inventory of Mars Surface Faults, Geophysical Research Abstracts, vol. 10, EGU2008-A-03574

Knapmeyer, M., Fischer, H.-H., Knollenberg, J., Seidensticker, K.J., Thiel, K., Arnold, W., Schmidt, W., Faber, C., Finke, F., Möhlmann, D. (2016). The SESAME/CASSE instrument listening to the MUPUS PEN

page 44 of 76,16352 words 
Seismic Moment Rate Estimation, v 2.0.3, last saved 08/01/2019 10:11:00

989

990

991

992

993

994

995

996

997

998

999

1000

1001

1002

1003

1004

1005

1006

1007

1008

1009

1010

1011

1012

1013

1014

1015

1016

1017

1018

1019

1020

1021

1022

1023

1024

1025

1026

1027

1028

insertion phase on comet 67P/Churyumov-Gerasimenko, Acta Atronautica, vol. 125, 234-249, doi: 10.1016/j.actaastro.2016.02.018

Knapmeyer, M., Oberst, J., Hauber, E., Wählisch, M., Deuchler, C., Wagner, R. (2006). Working models for spatial distribution and level of Mars' seismicity, J. Geophys. Res., vol. 111, E11006, doi:10.1029/2006JE002708

Knapmeyer-Endrun, B., Golombek, M. P., and Ohrnberger, M., 2017, Rayleigh wave ellipticity modeling and inversion for shallow structure at the proposed InSight landing site in Elysium Planitia, Mars: Space Science Reviews, v. 211, p. 339-382, DOI: 10.1007/s11214-016-0300-1

Ksanfomaliti, L.V., Zubkova, V.M., Morozov, N.A., Petrova, E.V. (1982), Microseisms at the Venera 13 and Venera 14 landing sites, Sov. Astron. Let. vol. 8, No., 4, Jul.-Aug. 1982, 241-242

Lognonné, P., W.T. Pike (2015). Planetary seismometry, in Extraterrestrial Seismology, ed. by V.C.H. Tong, R. Garcia, Cambridge University Press, Cambridge, pp. 36-48. Chap. 3. doi:10.1017/СBO9781107300668.006

Lognonné, P., and 180 co-authors, SEIS: The Seismic Experiment for Internal Structure of InSight, Space Science Reviews, submitted

Lorenz, R.D., Kedar, S., Murdoch, N., Lognonné, P., Kawamura, T., Mimoun, D., Banerdt, W.B. (2015). Seismometer Detection of Dust Devil Vortices by Ground Tilt., Bull. Seis. Soc. Am., vol. 105, No. 6, 30153023, doi: 10.1785/0120150133

Malin, M.C., Edgett, K.S., Posioolova, L.V., McColley, S.M., Noe Dobrea, E.Z. (2006), Present-Day impact cratering Rate and Contemporary Gully Activity on Mars, Science, vol. 314, 1573-1577, 10.1126/science.1135156

Morschhauser, A., Lesur, V., Grott, M. (2014) A spherical harmonic model of the lithospheric magnetic field of Mars, J. Geophys. Res. Planets, vol. 119, doi:10.1002/2013JE004555

Nakamura, Y. (2003) New identification of deep moonquakes in the Apollo lounar seismic data, Phys. Earth Planet. Int., vol. 139, 197-205, doi:10.1016/j.pepi.2003.07.017

Nakamura, Y. (2005) farside deep moonquakes and deep interior of the Moon, J. Geophys. Res., vol. 110, E01001, doi:10.1029/2004JE002332

Neukum, G., Jaumann, R., Hoffmann, H., Hauber, E., Head, J.W., Basilevsky, A.T., Ivanov, B.A., Werner, S.C., van Gasselt, S., Murray, J.B., McCord, T., \& the HRSC Co-Investigator Team(2004) recent and episodic volcanic and glacial activity on Mars revealed by the high resoltion stereo camera, Nature, vol. 432, 971-979

Oberst, J. (1987). Unusually high stress drops associated with shallow moonquakes, J. Geophys. Res., vol. 92, 1397-1405

Panning, M.P., Lognonné, P., Banerdt, W.B., Garcia, R., golombek, M., Kedar, S., Knapmeyer-Endrun, B., Mocuet, A., Teanby, N.A., Tromp, J., Weber, R., Beucler, E., Blanchette-Guertin, J.-F., Bozdağ, E., Drilleau, M., Gudkova, T., Hempel, S., Khan, A., Lekić, V., Murdoch, N., Plesa, A.-C., Rivoldini, A., Schmerr, N., Rua, Y., Verhoeven, O., Gao, C., Christensen, U., Clinton, J., Dehant, V., Giardini, D., Mimoun, D., Pike, W.T., Smrekar, S., Wiectorek, M., Knapmeyer, M., Wookey, J. (2017), Planned products of the Mars structure service for the InSight mission to Mars, Space Sci. Rev., vol. 211, 611-650, doi:10.1007/s11214-016-03175

page 45 of 76,16352 words 
1029 Panning, M.P., Beucler, E., Drilleau, M., Mocwuet, A., Lognonné, P, Banerdt, W.B. (2015) Verifying single1030 station seismic approaches using Earth-based data: preparation for data return from the InSight mission 1031 to Mars, larus, vol. 248, 230-242, doi:10.1016/j.icarus.2014.10.035

1032 Phillips, R.J. (1991). Expected rates of Marsquakes, in: Scientific Rationale and Requirements for a Global 1033 Seismic Network on mars, LPI Tech Rep. 91-02 LPI/TR-91-02, pp. 35-38, Lunar and Planet. Inst. Houston, 1034 Texas

1035 Plesa A.- C., Knapmeyer, M., Golombek, M.P., Breuer, D., Grott, M., Kawamura, T., Lognonné, P., Tosi, 1036 1037 N., and Weber, R. (2018), Present-day Mars' Seismicity Predicted from 3-D Thermal Evolution Models of Interior Dynamics, Geophys. Res. Lett., 45, doi:10.1002/2017GL076124.

1038 Reasenberg, P. (1985). Second-Order moment of central California seismicity, J. Geophys. Res., vol. 90, 1039 No. B7, 5479-5495

1040 Schulte, S.M.; Mooney, W.D. (2005) An updated global earthquake catalog for stable continental regions: 1041 reassessing the correlation with ancient rifts; Geophys. J. Int., vol. 161, 707-721

1042 Schulz, R.A., Okubo, C.H., Wilkins, S.J. (2006) Displacement-length scaling relations for faults on the ter1043 restrial planets, J. Struct. Geol. vol. 28, 2182-2193, doi: 10.1016/j.jsg.2006.03.034

1044 Schulz, R.A., Soliva, R., Okubo, C.H., Mège, D. (2010), Fault populations, in: Watters, T.R., Schulz, R.A. 1045 (eds.), Planetary Tectonics, Cambridge University Press, 457-510

1046 Siddiqi, A.A. (2002), Deep Space Chronicle, NASA SP-2002-4524, Monographs in Aerospace History, No. $1047 \quad 24,256$ pages

1048 Taylor, J., Teanby, N., Wookey, J. (2013). Estimates of seismic activity in the Cerberus Fossae region on 1049 Mars, vol. 118, 2570-2581, doi:10.1002/2013JE004469

1050 Teanby, N.A., Wookey, J. (2011), Seismic detection of meteorite impacts on Mars, Phys. Earth Planet. 1051 Int., vol. 186, 70-80, doi:10.1016/j.pepi.2011.03.004

1052 Toksöz, M. Nafi (1979). Planetary Seismology and Interiors, Rev. Geoph. Space Phys., vol. 17, No. 7, 164110531655

1054 Vere-Jones, D. (1976). A branching model for crack propagation, Pure Appl. Geophys., vol. 114, 711-725

1055 Vere-Jones, D., Robinson, R., Yang., W. (2001). Remarks on the accelerated moment release model: 1056 problems of model formulation, simulation and estimation; GJ, vol. 144, 517-531

1057 Vostreys, R.W. (1980), Data User's Note - Apollo Seismological Investigations, NSSDC/WDC-A-R\&S 80-11, 1058 1059 NASA-TM-82280, 27 pages, available online: https://ntrs.nasa.gov/archive/nasa/casi.ntrs.nasa.gov/19810009447.pdf, last accessed 19.02.2018

1060 Wason, H.R., Das, R., Sharma, M.L. (2012) Magnitude conversion problem using general orthogonal re1061 gression, Geophys. J. Int., vol. 190, 1091-1096, doi: 10.1111/j.1365-246X.2012.05520.x

1062 Weber, R.C.; Bills, B.G., Johnson, C.L. (2010) A simple physical model for deep moonquake occurrence 1063 times, Phys. Earth Planet. Int., vol. 182, 152-160, doi:10.1016/j.pepi.2010.07.009

1064 Weisstein, Eric W. (undated). Gamma Function, MathWorld--A Wolfram Web Resource. 1065 http://mathworld.wolfram.com/GammaFunction.html, last accessed 26. October 2017

1066 Wyrick, D. Y., Morris, A.P., Ferrill, D.A. (2018), Normal fault gropth in analog mdoels and on Mars, Icarus, 1067 vol. 212, 559-567, doi:10.1016/j.icarus.2011.01.011 
1068 Young, J.B.; Presgrave, B.W.; Aichele, H.; Wiens, D.A.; Flinn, E.A. (1996) The Flinn-Engdahl Regionalisation 1069 Scheme: the 1995 revision; Phys. Earth Planet. Int., vol. 96, 223-297

1070 Zhuang, J., D. Harte, M.J. Werner, S. Hainzl, and S. Zhou (2012), Basic models of seismicity: temporal 1071 models, Community Online Resource for Statistical Seismicity Analysis, doi:10.5078/corssa-79905851. 1072 Version 1.0, issued 14. Aug. 2012. Available at http://www.corssa.org

1073 Zhuang, J. \& S. Touati (2015), Stochastic simulation of earthquake catalogs, Community Online Resource 1074 for Statistical Seismicity Analysis, doi:10.5078/corssa-43806322. Version 1.0, issued 19. March 2015, 1075 Available at http://www.corssa.org

1076 Zuber, M.T., Solomon, S.C., Phillips, R.J., Smith, D.E., Tyler, G.L., Aharonson, O., Balmino, G., Banerdt, 1077 W.B., Head, J.W., Johnson, C.L., Lemoine, F.G., McGovern, P.J., Neumann, G.A., Rowlands, D.G., Zhong, S. 1078 (2000) Internal structure and early thermal evolution of Mars from Mars Global Surveyor Topography 1079 and Gravity, Science, vol. 287, 1788-1793 
Seismic Moment Rate Estimation, v 2.0.3, last saved 08/01/2019 10:11:00

\begin{tabular}{|c|c|}
\hline 1081 & DLR Institute for Planetary Research \\
\hline 1082 & Rutherfordstr. 2 \\
\hline 1083 & 12489 Berlin \\
\hline 1084 & Germany \\
\hline 1085 & (MK, ACP) \\
\hline 1086 & Max Planck Institute for Solar System Research \\
\hline 1087 & Justus-von-Liebig-Weg 3 \\
\hline 1088 & 37077 Göttingen \\
\hline 1089 & Germany \\
\hline 1090 & (BKE) \\
\hline 1091 & Jet Propulsion Laboratory \\
\hline 1092 & California Institute of Technology \\
\hline 1093 & Pasadena, CA 91109 \\
\hline 1094 & USA \\
\hline 1095 & (MPG, SK) \\
\hline 1096 & NASA Marshall Space Flight Center \\
\hline 1097 & 320 Sparkman Drive \\
\hline 1098 & Huntsville \\
\hline 1099 & AL 35805 \\
\hline 1100 & USA \\
\hline 1101 & $(\mathrm{RCW})$ \\
\hline 1102 & Swiss Seismological Service \\
\hline 1103 & ETH Zürich \\
\hline 1104 & Sonneggstrasse 5 \\
\hline 1105 & 8092 Zürich \\
\hline 1106 & Switzerland \\
\hline 1107 & (MB, JFC, SS) \\
\hline
\end{tabular}


Seismic Moment Rate Estimation, v 2.0.3, last saved 08/01/2019 10:11:00

\begin{tabular}{|c|c|}
\hline 1108 & AWE Blacknest \\
\hline 1109 & Brimpton \\
\hline 1110 & Reading \\
\hline 1111 & Berkshire RG7 4RS \\
\hline 1112 & UK \\
\hline 1113 & (JS) \\
\hline 1114 & School of Earth Sciences \\
\hline 1115 & University of Bristol \\
\hline 1116 & Wills Memorial Building \\
\hline 1117 & Queens Road \\
\hline 1118 & Bristol BS8 1RJ \\
\hline 1119 & UK \\
\hline 1120 & (NAT) \\
\hline 1121 & Institute de Physique du Globe de Paris (IPGP) \\
\hline 1122 & Bât. Lamarck \\
\hline 1123 & 35 rue Hélène Brion \\
\hline 1124 & 75205 Paris CEDEX 13 \\
\hline 1125 & France \\
\hline 112 & $(\mathrm{TK}, \mathrm{CP}, \mathrm{PL})$ \\
\hline
\end{tabular}

1127 


\begin{tabular}{|c|c|c|c|c|}
\hline Planet & Reference & Method & $\begin{array}{l}\text { Annual Rate } \\
{[\mathrm{Nm} / \mathrm{yr}]}\end{array}$ & $\begin{array}{l}\text { Annual Rate } \\
\text { [Equiv. Mag. } \\
\text { per yr] }\end{array}$ \\
\hline Earth & GCMT & $\begin{array}{l}\text { observed quakes, 1976- } \\
2018\end{array}$ & $7.61 \times 10^{21}$ & 8.5 \\
\hline Moon & Oberst, 1987 & observed HFT quakes & $7.27 \times 10^{14}$ & 3.8 \\
\hline \multirow[t]{4}{*}{ Mars } & Anderson et al., 1977 & $\begin{array}{l}\text { Viking non-observation } \\
\text { of quakes }\end{array}$ & $\begin{array}{l}\text { Moon } \leq \text { Mars } \\
<\text { Earth }\end{array}$ & \\
\hline & $\begin{array}{l}\text { Goins \& Lazarewicz, } \\
1979\end{array}$ & $\begin{array}{l}\text { Viking non-observaation } \\
\text { of quakes }\end{array}$ & $<$ Earth & \\
\hline & Phillips, 1991 & $\begin{array}{l}\text { 1D lithospheric cooling } \\
\text { model }\end{array}$ & $4.8 \times 10^{18}$ & 6.4 \\
\hline & $\begin{array}{l}\text { Golombek et al., } \\
1992\end{array}$ & $\begin{array}{l}\text { Observed surface fault- } \\
\text { ing }\end{array}$ & $10^{18}$ & 5.9 \\
\hline
\end{tabular}


Seismic Moment Rate Estimation, v 2.0.3, last saved 08/01/2019 10:11:00

\begin{tabular}{|l|l|l|l|l|}
\hline Golombek, 2002 & Observed surface fault- & $10^{18}$ to $10^{19}$ & 5.9 to 6.6 \\
ing & Knapmeyer et al., & 1D lithospheric cooling & $3.42 \times 10^{16}$ & 4.96 \\
2006 & model, weak, medium, & $5.99 \times 10^{17}$ & 5.8 \\
\hline Taylor et al., 2013 & Observed surface fault- & $>5.5 \times 10^{16}$ & $>5.09$ \\
& ing scenario & $4.78 \times 10^{18}$ & 6.4 \\
\hline Plesa et al., 2018 & 3D whole-mantle ther- & $5.7 \times 10^{16}$ to & 5.1 to 7 \\
\hline
\end{tabular}

1132 Equivalent magnitudes are moment magnitudes computed according to eq. 1 assuming that the en1133 tire moment release of one year is put into a single event. 
Seismic Moment Rate Estimation, v 2.0.3, last saved 08/01/2019 10:11:00

Table 2 Quality levels used to categorize Goldstone seismograms

\begin{tabular}{|l|l|l|}
\hline Category & Definition & Event count \\
\hline A & prominent arrivals of various body and surface wave phases & 38 \\
\hline B & P arrival and Rayleigh waves clearly above noise level & 19 \\
\hline C & P arrival and Rayleigh waves barely above noise level & 13 \\
\hline D & Only body waves visible & 4 \\
\hline E & Only surface waves visible & 60 \\
\hline F & Invisible & 170 \\
\hline
\end{tabular}


Seismic Moment Rate Estimation, v 2.0.3, last saved 08/01/2019 10:11:00

Table 3 Annual moment rate estimations for different settings on Earth

\begin{tabular}{|c|c|c|c|c|}
\hline Subset (No. of events) & $\Sigma$ & $K S_{10}$ & $N L V R$ & Units \\
\hline \multirow[t]{2}{*}{ All events (49178) } & 76.1 & 54.9 & 33.6 & $10^{20} \mathrm{Nm} / \mathrm{yr}$ \\
\hline & 8.52 & 8.43 & 8.28 & Magnitude \\
\hline \multirow[t]{2}{*}{ Subduction Zones } & 66.9 & 54.9 & 33.6 & $10^{20} \mathrm{Nm} / \mathrm{yr}$ \\
\hline & 8.48 & 8.43 & 8.28 & Magnitude \\
\hline \multirow[t]{2}{*}{ Mid-Ocean Ridges } & 1.63 & 1.11 & 1.07 & $10^{20} \mathrm{Nm} / \mathrm{yr}$ \\
\hline & 7.41 & 7.3 & 7.29 & Magnitude \\
\hline \multirow{2}{*}{$\begin{array}{l}\text { Subduction Zones, } \\
z \geq 100 \mathrm{~km}(7297)\end{array}$} & 7.38 & 6.1 & 2.49 & $10^{20} \mathrm{Nm} / \mathrm{yr}$ \\
\hline & 7.85 & 7.79 & 7.53 & Magnitude \\
\hline \multirow{2}{*}{$\begin{array}{l}\text { Subduction Zones, } \\
z<100 \mathrm{~km}(26003)\end{array}$} & 59.8 & 52.1 & 33.6 & $10^{20} \mathrm{Nm} / \mathrm{yr}$ \\
\hline & 8.45 & 8.41 & 8.28 & Magnitude \\
\hline \multirow{2}{*}{$\begin{array}{l}\text { Stable Continental Re- } \\
\text { gions, historic events }\end{array}$} & 0.0571 & 0.0405 & 0.0415 & $10^{20} \mathrm{Nm} / \mathrm{yr}$ \\
\hline & 6.44 & 6.34 & 6.35 & Magnitude \\
\hline \multirow[t]{2}{*}{ Stable Continental Re- } & 0.101 & 0.124 & 0.17 & $10^{20} \mathrm{Nm} / \mathrm{yr}$ \\
\hline & 6.6 & 6.66 & 6.75 & Magnitude \\
\hline
\end{tabular}


Seismic Moment Rate Estimation, v 2.0.3, last saved 08/01/2019 10:11:00

1139 Annual moment rate estimations for several subsets of the GCMT catalog from 1976 to 2018, for histori-

1140 cally witnessed events in stable continental regions between 1500 and 1949, and finally instrumentally

1141 recorded events in stable continental regions, between 1950 and 2003. The first column is the estimate

1142 resulting from summing up all events in the subset, the second is the KS for the 10 strongest events, and

1143 the third column is the NLVR estimator resulting from the single strongest event. Each estimate is given

1144 in Newton meters per year and as moment magnitude of a single event releasing the same amount of

1145 Newton meters.

1146 
Seismic Moment Rate Estimation, v 2.0.3, last saved 08/01/2019 10:11:00

1147

Table 4 Annual moment rate models for Mars

\begin{tabular}{|l|c|c|c|c|}
\hline Model & $\dot{M}_{\boldsymbol{S}}[\mathrm{Nm} / \mathbf{y r}]$ & $\boldsymbol{M}_{\boldsymbol{M a x}}[\mathrm{Nm}]$ & $\boldsymbol{M}_{\boldsymbol{C}}[\mathrm{Nm}]$ & $\boldsymbol{m}_{\boldsymbol{S}}-\boldsymbol{m}_{\boldsymbol{C}}$ \\
\hline StrongFew & $4.78 \times 10^{18}$ & $3.36 \times 10^{20}$ & $1.31 \times 10^{20}$ & -0.96 \\
\hline StrongMany & $4.78 \times 10^{18}$ & $3.42 \times 10^{16}$ & $1.33 \times 10^{16}$ & +1.70 \\
\hline Medium & $5.99 \times 10^{17}$ & $2.41 \times 10^{18}$ & $9.42 \times 10^{17}$ & -0.13 \\
\hline WeakMany & $3.42 \times 10^{16}$ & $3.42 \times 10^{16}$ & $1.33 \times 10^{16}$ & +0.27 \\
\hline WeakFew & $3.42 \times 10^{16}$ & $3.37 \times 10^{20}$ & $1.32 \times 10^{20}$ & -2.39 \\
\hline
\end{tabular}

1150 models of Knapmeyer et al., 2006. All models use a slope parameter of $\boldsymbol{\beta}=\mathbf{0 . 6 2 5}$. 
Seismic Moment Rate Estimation, v 2.0.3, last saved 08/01/2019 10:11:00

1152

page 56 of 76,16352 words 


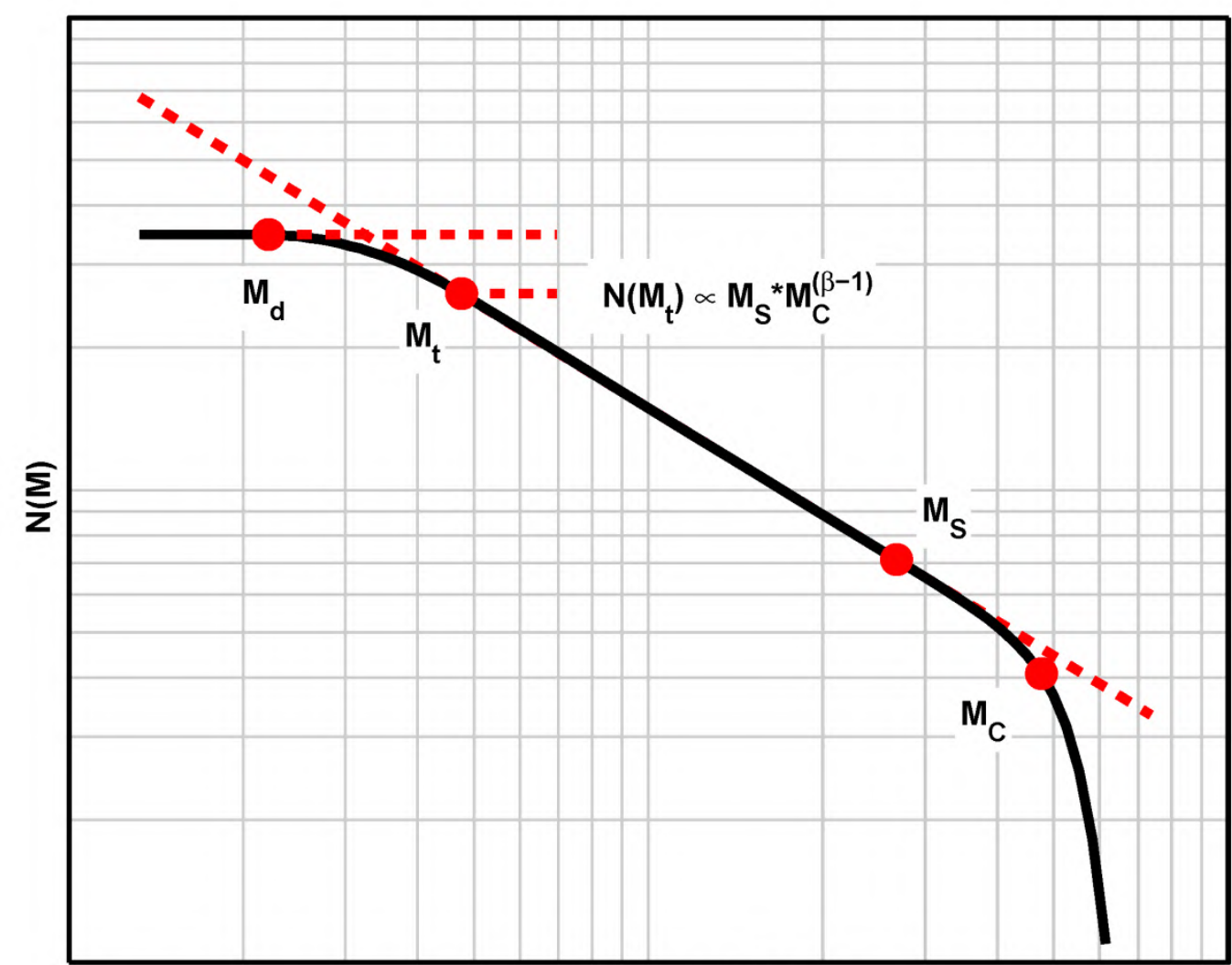

Seismic Moment [Nm]

Figure 1 Schematic depiction of the tapered Gutenberg-Richter distribution in survivor form, showing the number $N$ of events exceeding moment $\boldsymbol{M}$. Bullets mark characteristic moment values: $\boldsymbol{M}_{\boldsymbol{d}}$ - detection threshold, no event smaller than this is contained in a catalog; $M_{t}$ - completeness threshold, all events above this are cataloged; $M_{S}$ - size of an event corresponding to the average moment rate; $M_{C}$ - corner moment. The corner moment may or may not be larger than $M_{S}$. slanted dashed line: prediction according to a Gutenberg-Richter law with the same slope. Horizontal dashed lines: (lower) number of 
Seismic Moment Rate Estimation, v 2.0.3, last saved 08/01/2019 10:11:00

1164

page 58 of 76,16352 words 


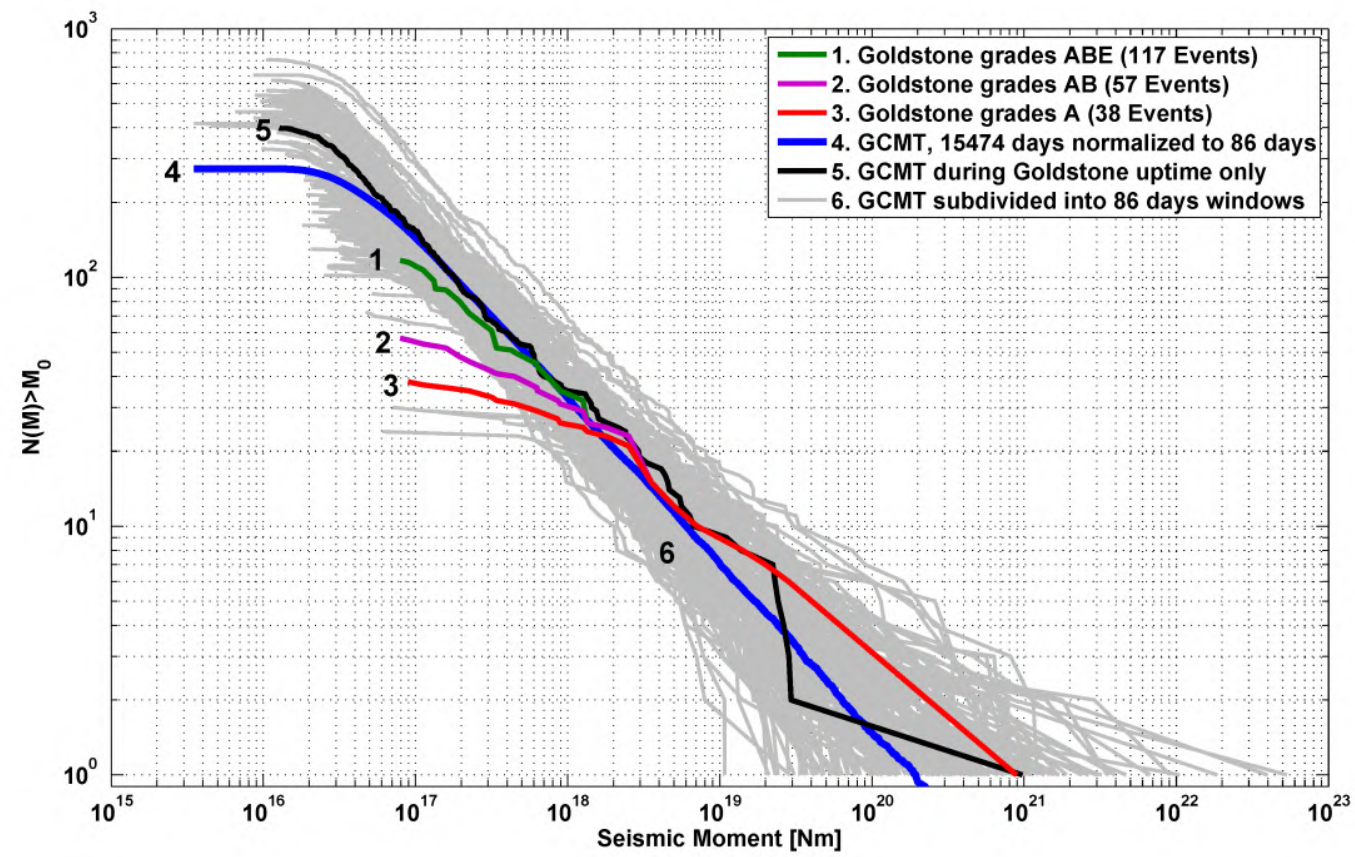

Figure 2 Size-frequency survivor distributions for events detected at Goldstone (in groups containing grade A only, or grades $A$ and $B$, or grades A, B, and E, as indicated in legend. Note that the $A B E$ line is partially covered by the $A B$ line, which

1169 in turn gets covered by the A line), the subset of events listed in the GCMT for the uptime of the Goldstone experiment (la-

1170 beled 5), the whole GCMT scaled down to a duration of 86 days (labeled 4). The gray curves in the background show distribu-

1171 tions for consecutive 86-days time windows (i.e. days 1 to 86,87 to 173,174 to 260 , ... etc.) throughout the entire GCMT catalog. 
Seismic Moment Rate Estimation, v 2.0.3, last saved 08/01/2019 10:11:00

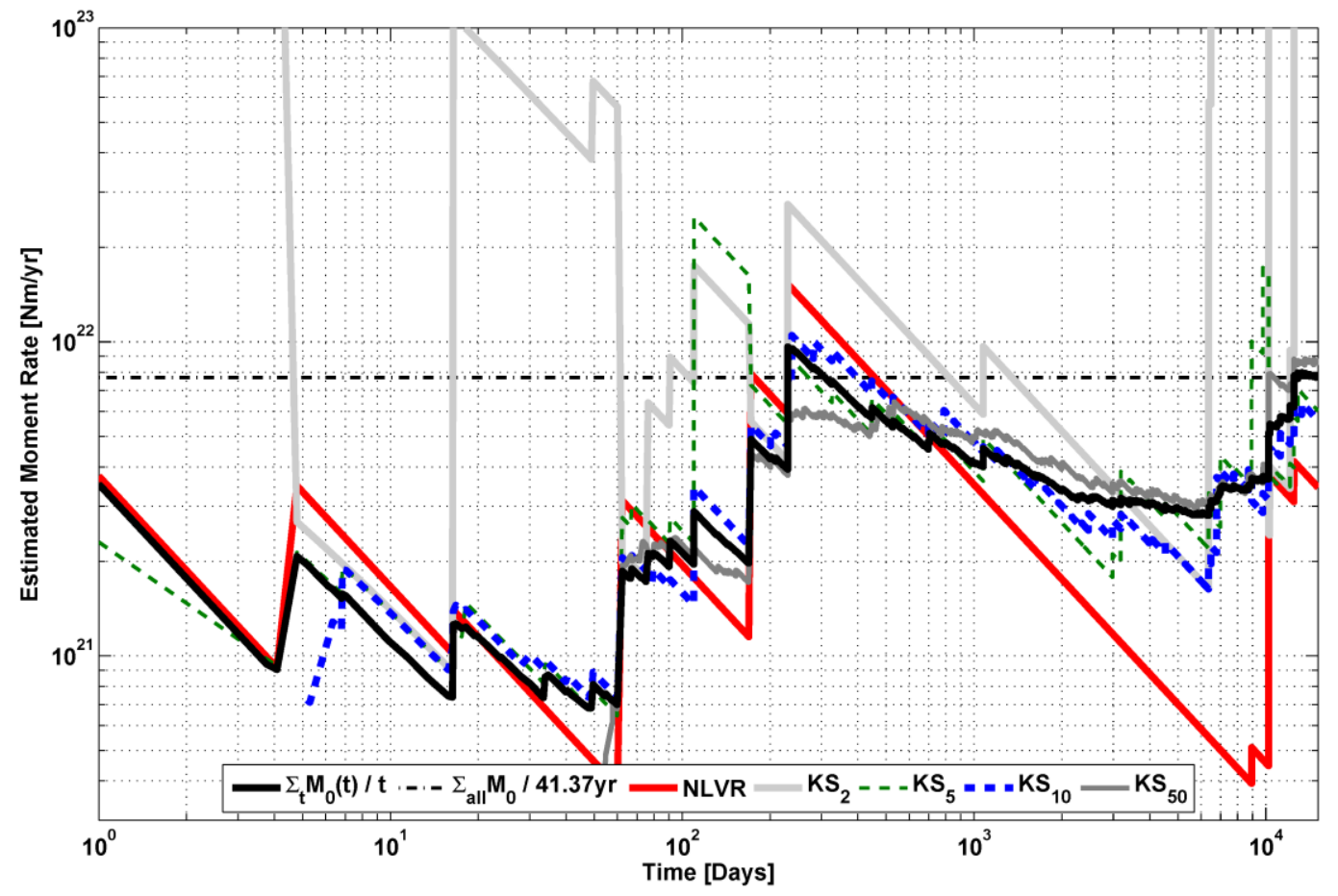

1174

1175

Figure 3 Evolution in time of moment rate estimations for Earth. The bold black curve is based on summation of all events

1176 in the GCMT catalog. The horizontal line marks its value at the end of the cataloged time (01.01.1977 to 18.05.2018). Other

1177 lines show time series for the $N L V R$ and $K S_{k}$ estimators according to the legend. 
Seismic Moment Rate Estimation, v 2.0.3, last saved 08/01/2019 10:11:00

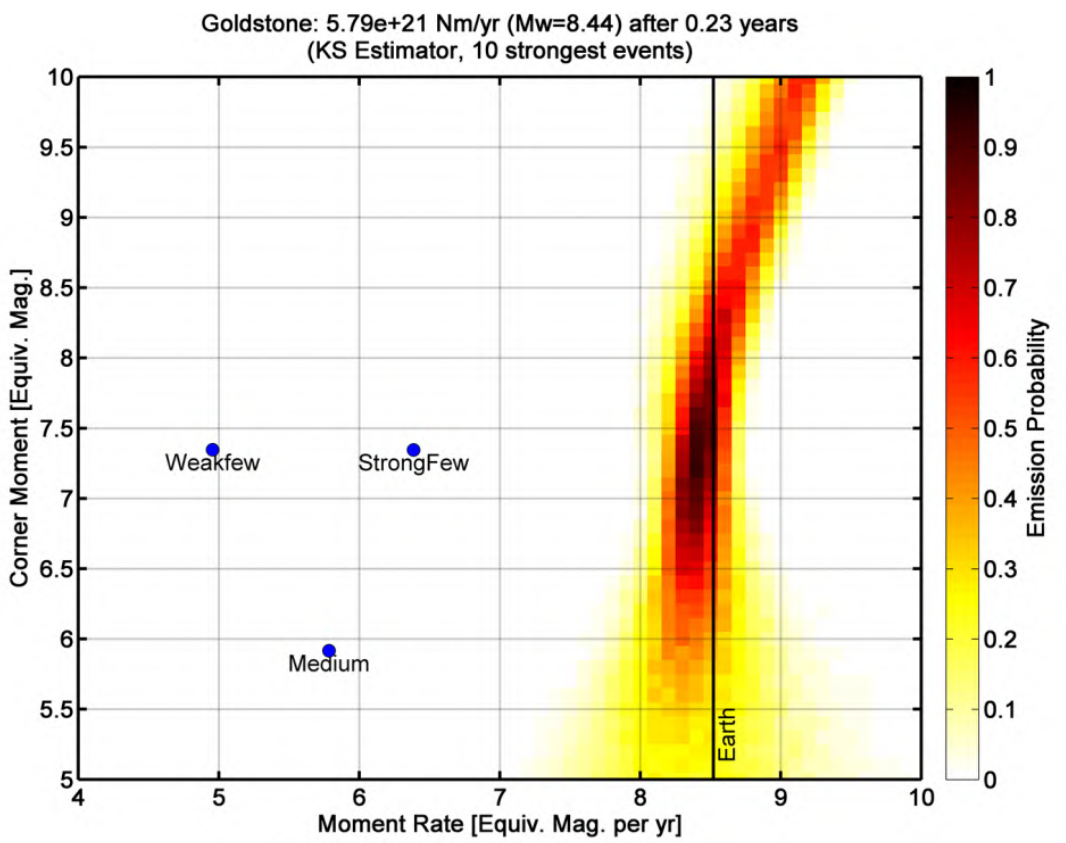

Figure $4 \mathrm{KS}_{10}$ emission probability for the events detected at Goldstone (grade A events only). Dots mark the moment

1181 rates and corner moments of all five Mars seismicity scenarios of Knapmeyer et al. (2006), pixel color encodes the emission Earth, as listed in Table 1. 


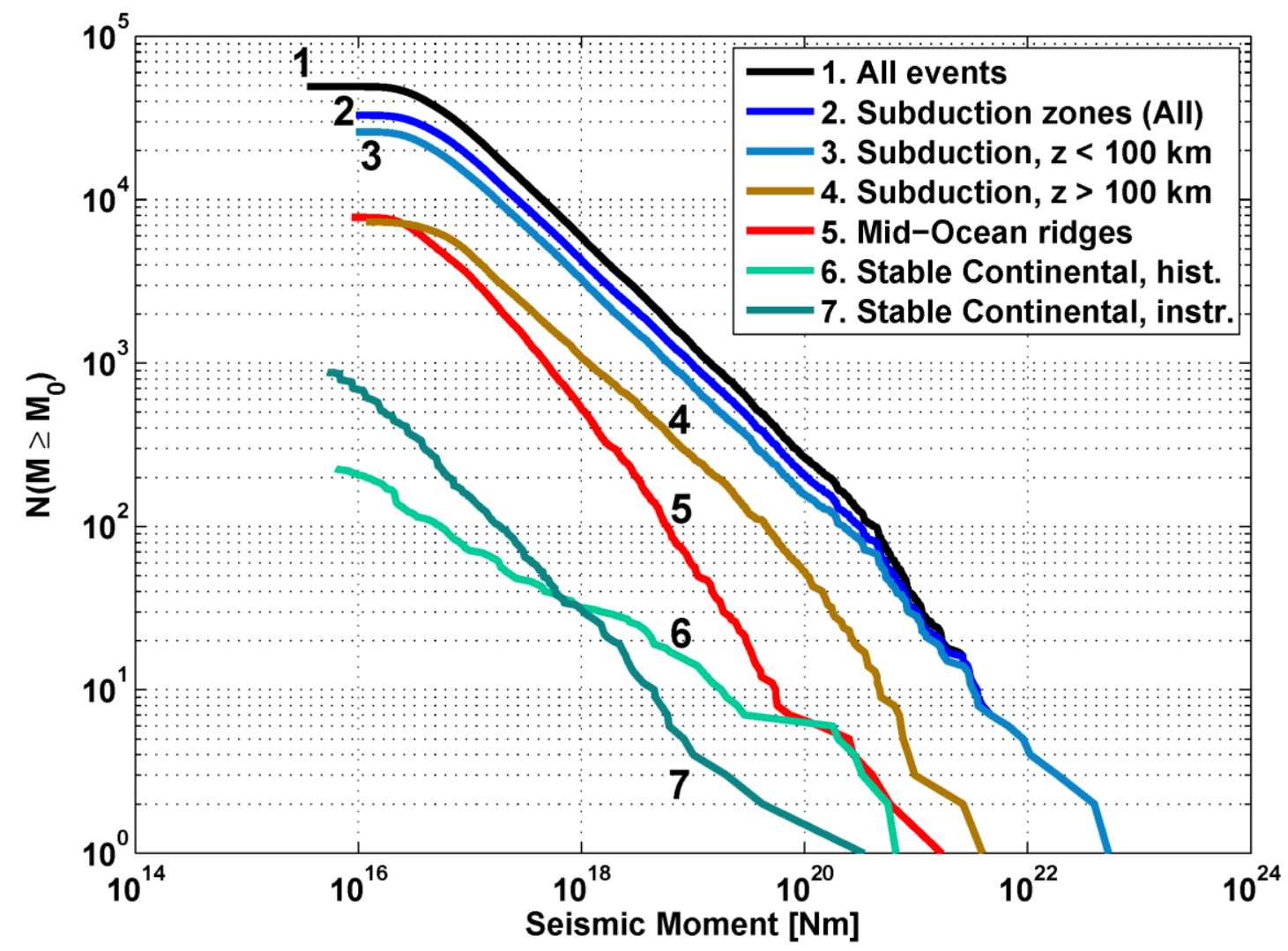

1187 Figure 5 Size-Frequency distributions of several subsets of the GCMT catalog and of the Stable Continen-

1188 tal Regions catalog. Subduction zones and mid-ocean ridges are identified by using the Flinn-Engdahl

1189 regionalization scheme, and subduction zone events are further subdivided into events occurring above

1190 and below $100 \mathrm{~km}$ depth. The complete GCMT catalog is shown for comparison. For the Stable Continen-

1191 tal Regions catalog, the distributions of events from 1500 to 1949 and from 1950 to 2003 are shown

1192 separately. 


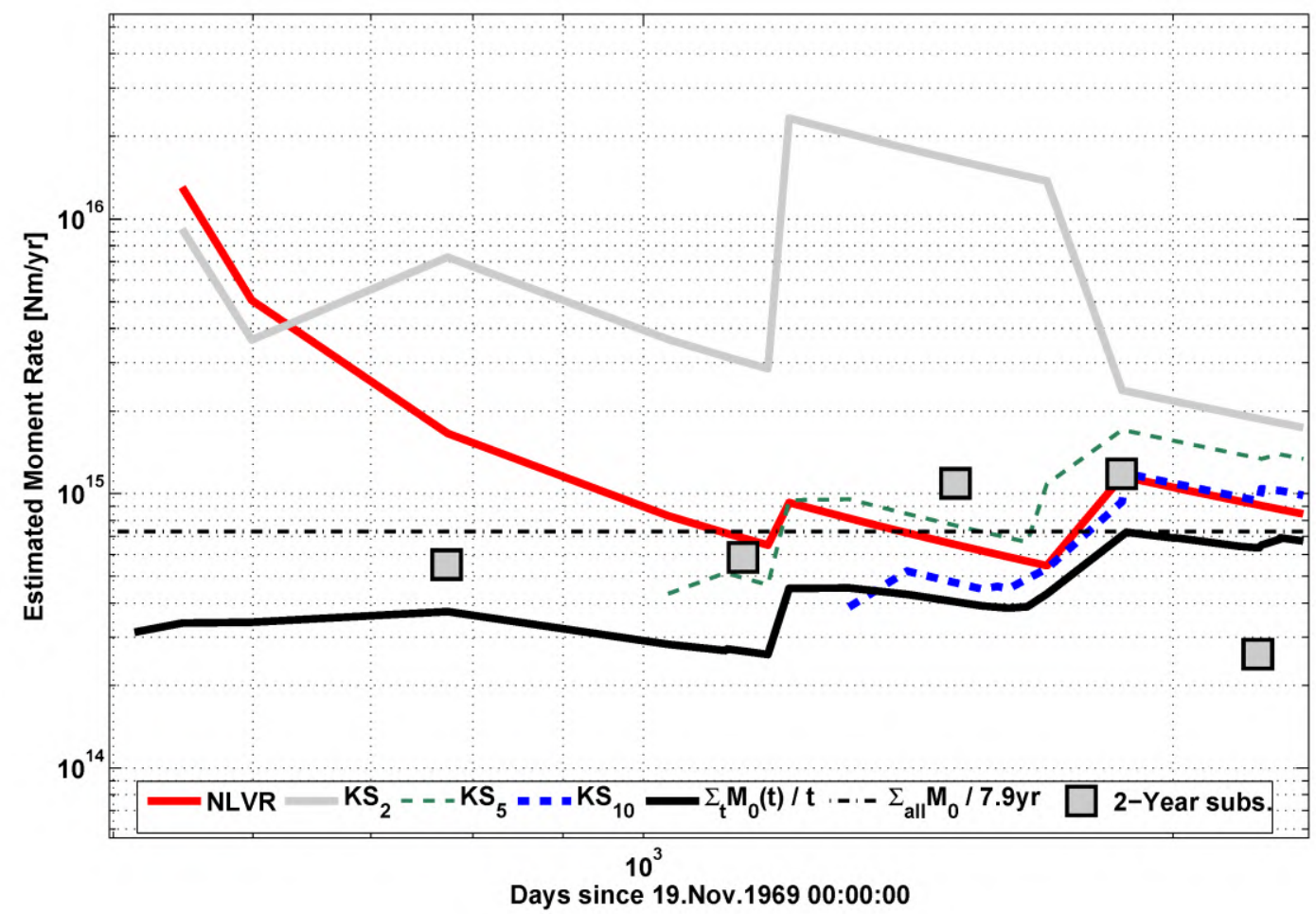

1195

Figure 6 Evolution in time of moment rate estimations for shallow moonquakes. Lines denote estimator output as in Figure 3, applied to the seismic moments of Oberst (1987). Squares denote the sum of two calendar years of registrations, plot- 
Seismic Moment Rate Estimation, v 2.0.3, last saved 08/01/2019 10:11:00

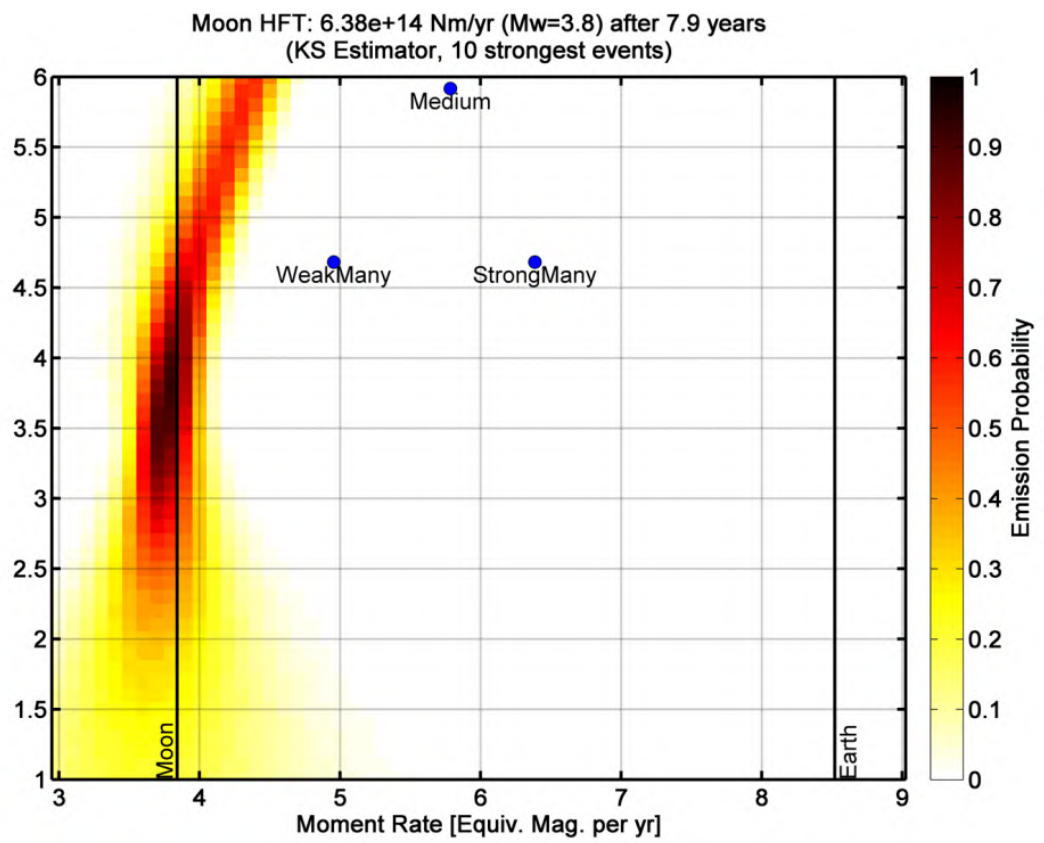

Figure $7 \mathrm{KS}_{10}$ emission probability for shallow moonquakes. Dots mark the moment rates and corner moments of Mars 1203 seismicity scenarios of Knapmeyer et al. (2006) for comparison, pixel color encodes the emission probability for matching the target rate within 0.2 magnitudes, as derived from 1000 simulated catalogs per pixel. Coordinate axes are labeled in terms of equivalent moment magnitudes. The two vertical black lines give the moment rates of Moon and Earth, as listed in Table 1. 
Seismic Moment Rate Estimation, v 2.0.3, last saved 08/01/2019 10:11:00

1207

1208

1209

page 65 of 76,16352 words 


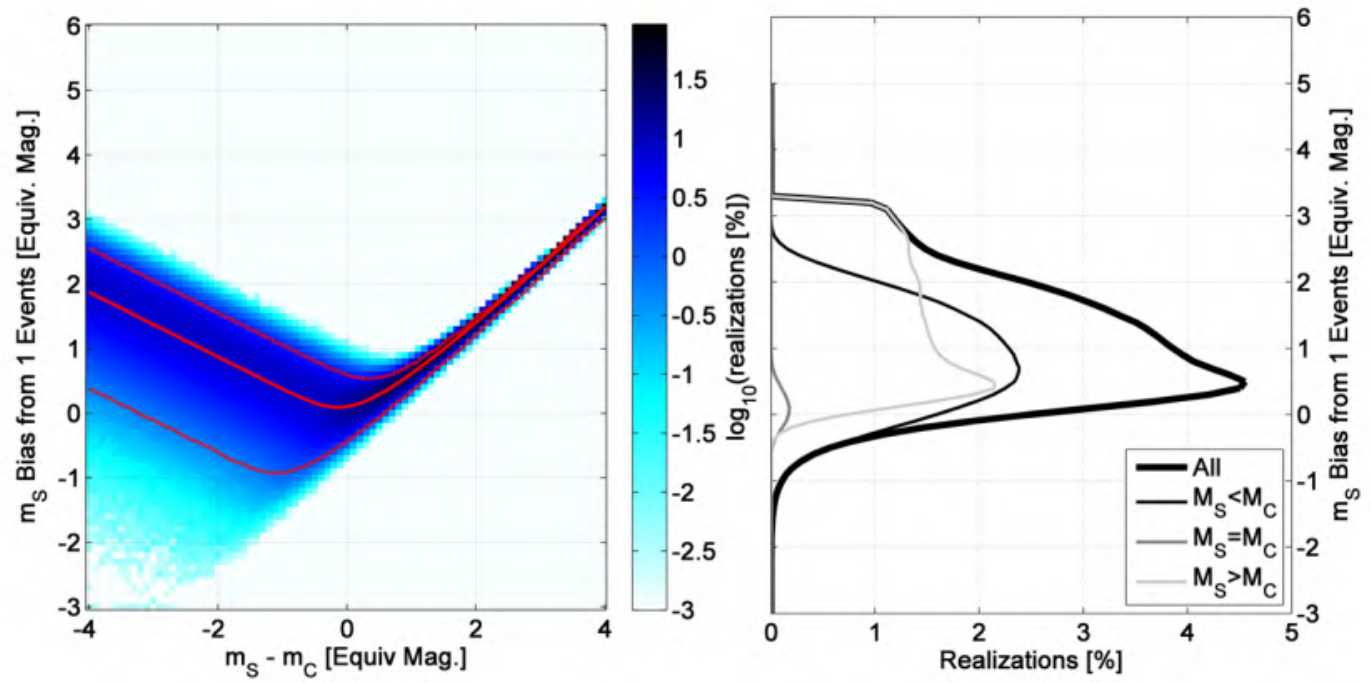

1213 catalogs. Left: Histograms resulting from the 8108910 synthetic catalogs generated for the cumulative moment to corner

1214 moment ratio indicated on the x-axis, shading encodes the logarithm of the percentage of realizations in each moment rate

1215 bias bin of width 0.1. Positive values on the $y$-axis indicate underestimation. Solid line: median, dashed lines: upper and

1216 lower boundary of the 95\%-quantile centered around the median. Right: marginal distribution density resulting from row-

1217 wise integration of the figure to the left. Thick line: all catalogs, other: relation between moment rate $\mathrm{M}_{\mathrm{S}}$ and corner moment $M_{C}$ as indicated in legend. 
Seismic Moment Rate Estimation, v 2.0.3, last saved 08/01/2019 10:11:00
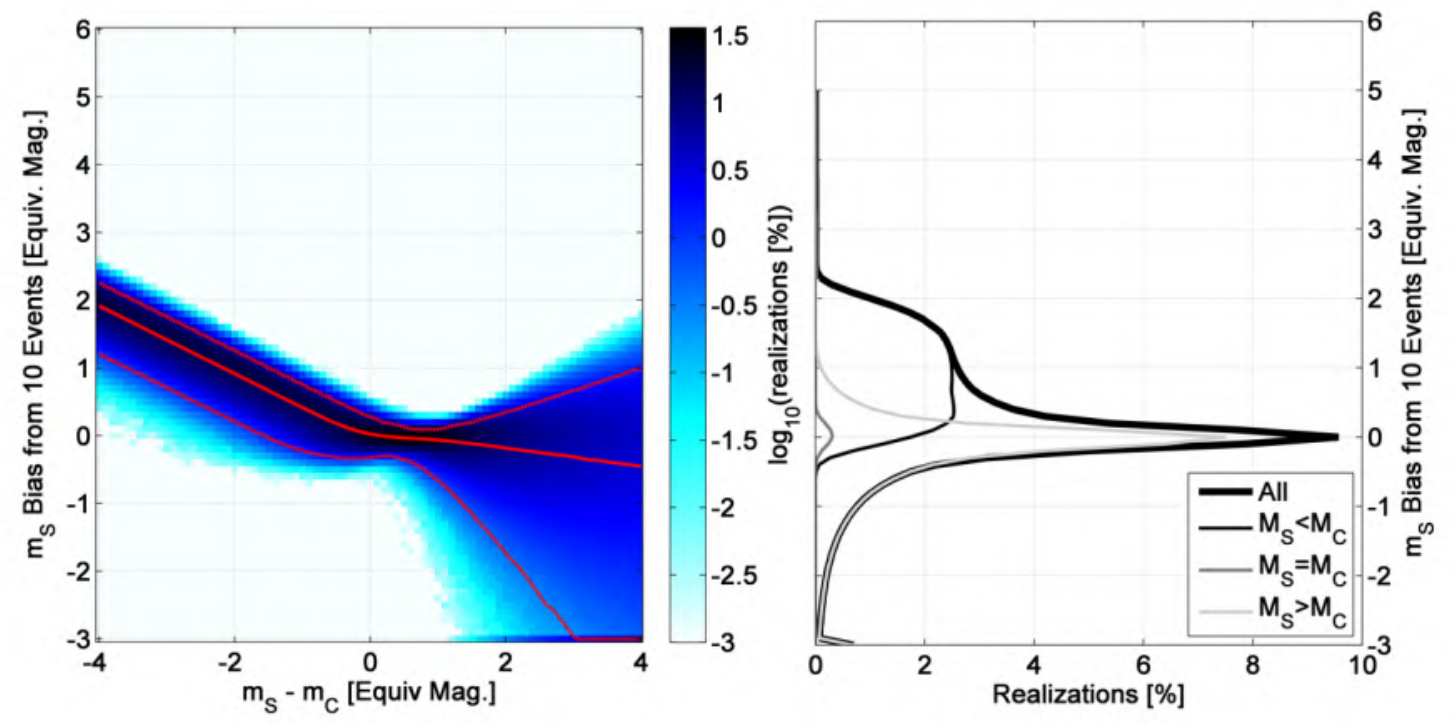

1220

1221 Figure $9 \mathrm{KS}_{10}$ Cumulative moment bias for synthetic catalogs, using $\beta=2 / 3$ in both generation and interpretation of catalogs. See Figure 8 for a description of graph elements. 

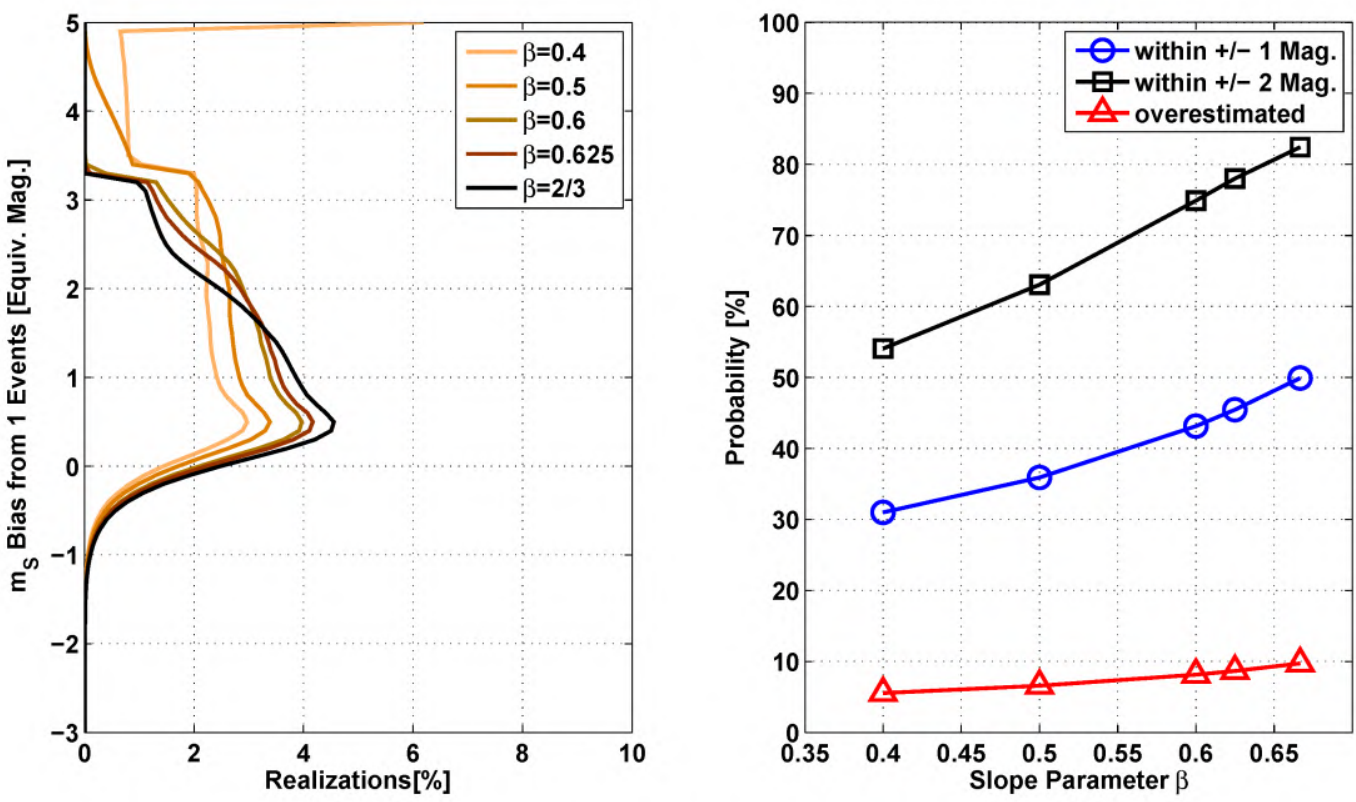
and the fraction of overestimated moment rates (red). 

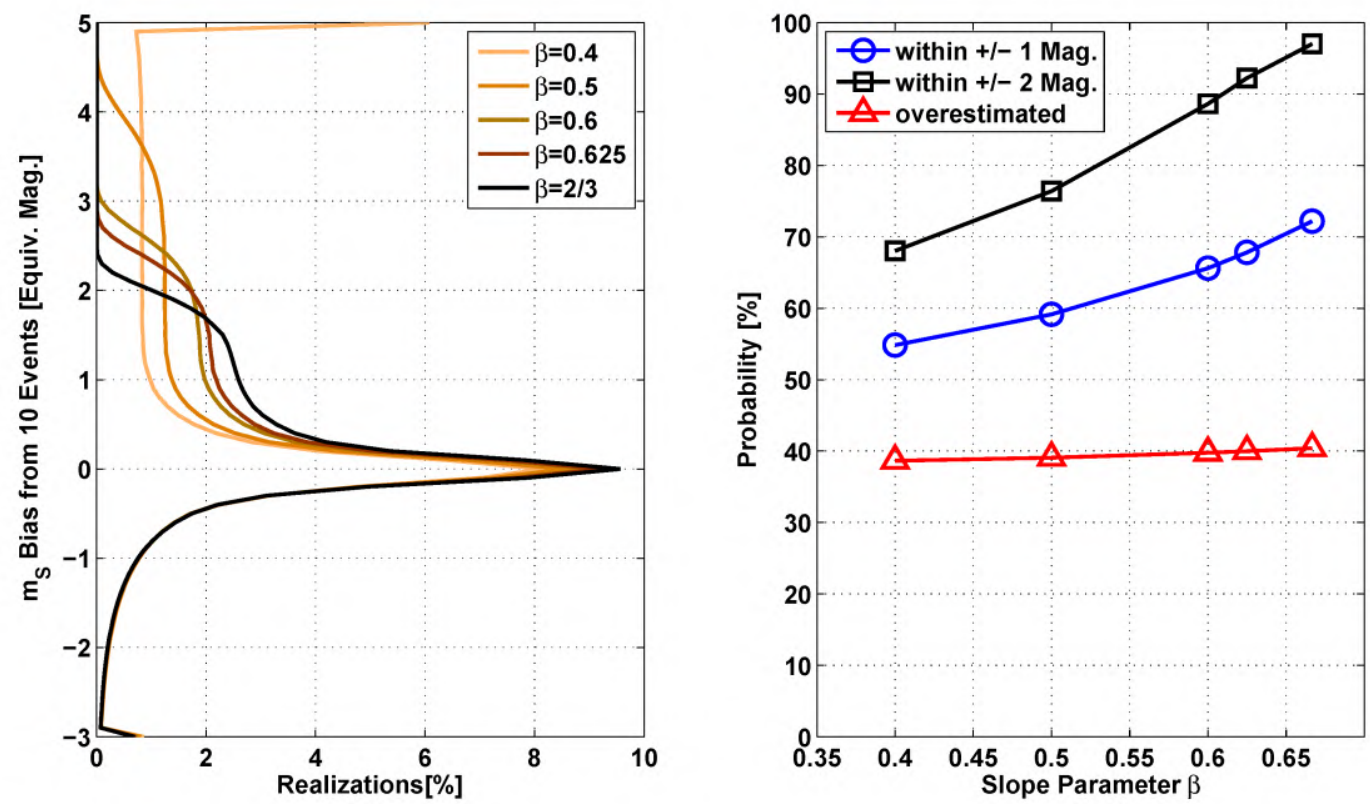

1232

1233

Figure $11 \mathrm{KS}_{10}$ estimator marginal distribution densities for different slope parameters. See Figure 10 for a plot descrip1234 tion

1235 

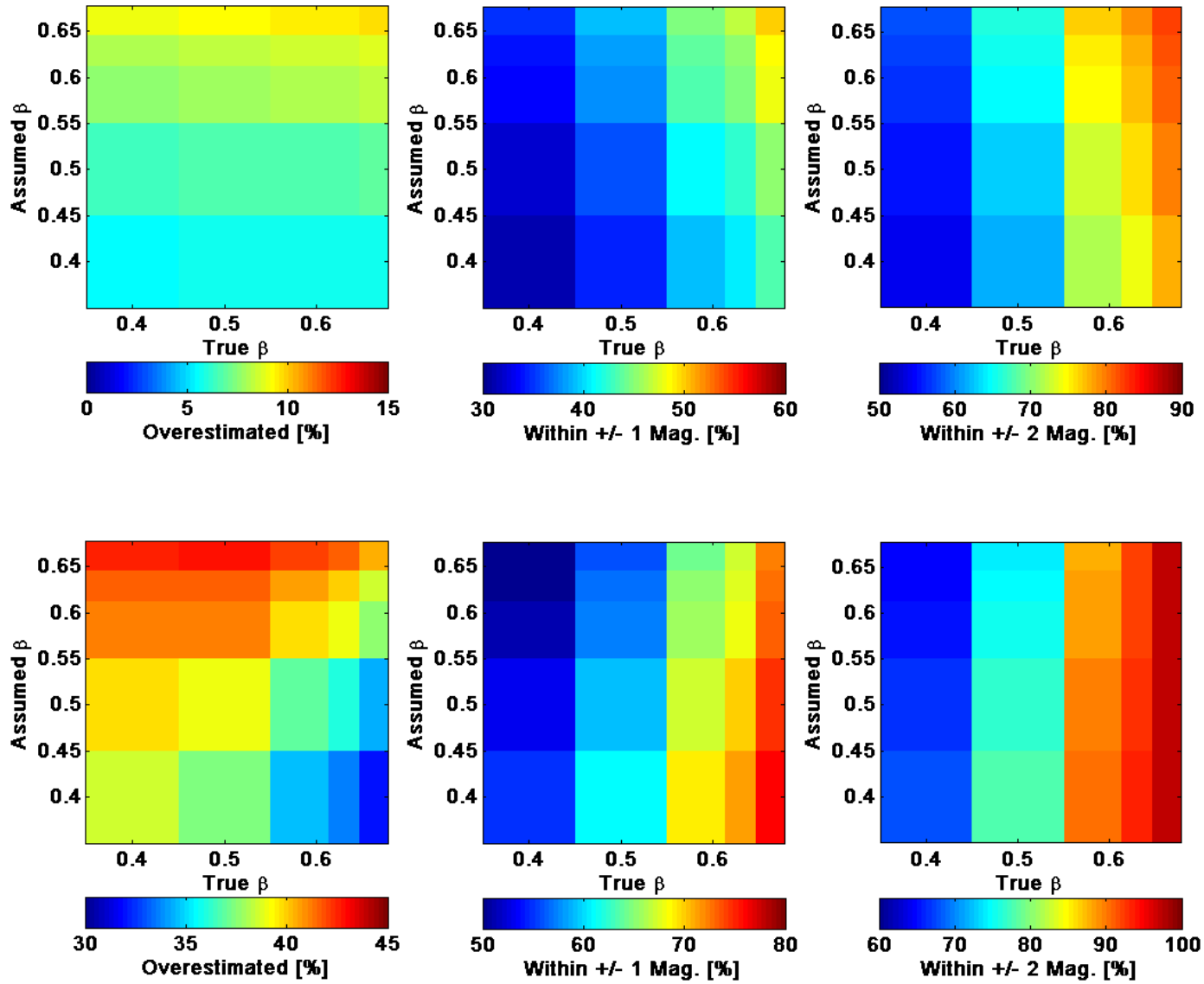

Figure 12 Distribution width parameters for NLVR (top row) and $K S_{10}$ (bottom row) estimators. Each uniformly colored patch indicates the width parameter of distribution densities as shown in Figure 10 and Figure 11. True slope and slope as-

1239 sumed during catalog evaluation were taken from $\{0.4,0.5,0.6,0.625,2 / 3\}$ to form a square matrix of test cases. From left

1240 to right: percentage of cases with overestimated moment rate, percentage of moment rate estimations within \pm 1 magnitude

1241 of true value, or within \pm 2 magnitudes, respectively. Panels in the same subplot column share the same color range width,

1242 although the absolute values differ. 

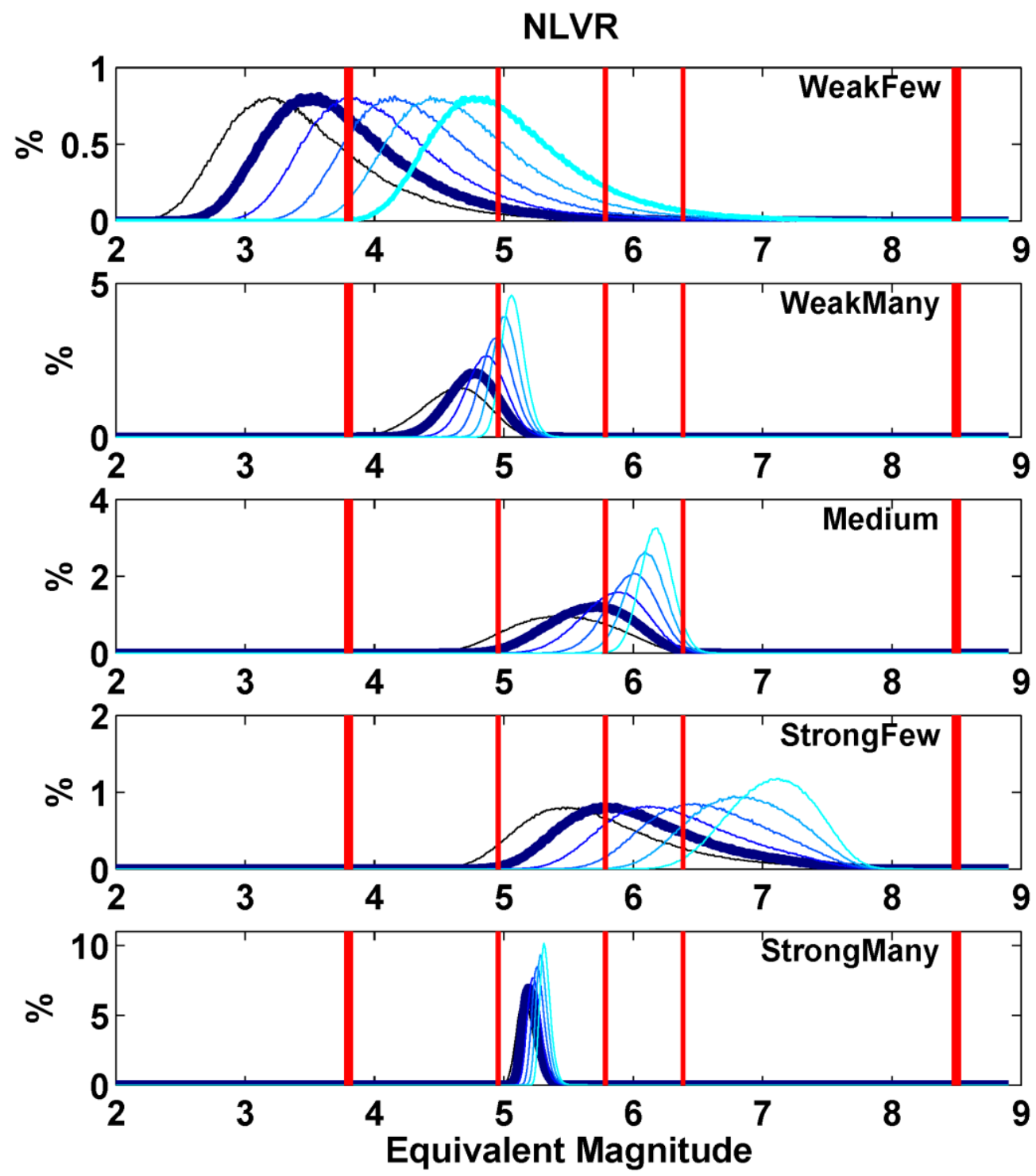

Figure 13 Distribution of NLVR estimators for Mars-specific seismicity models of Knapmeyer et al. (2006) and registration

1246 times of $1,2,4,8,16$, and 32 years (envelopes of histograms with bin width 0.01 magnitudes, scaled to percents). Panels from

top to bottom show the distributions for seismicity models as indicated in the panel. Note the different y axis ranges.. The

1248 seismic moment rate is given as the moment magnitude of a single event that releases the entire moment in one event (the

1249 axis range corresponds to $1.3 \times 10^{12} \ldots 4 \times 10^{22} \mathrm{Nm}$ ). Darker hues correspond to shorter registration times, the two-year 1250 case also uses a thicker line. Vertical lines from left to right denote moment rates (as equivalent magnitudes) for the Moon, 1251 both Weak scenarios, Medium scenario, both Strong scenarios, and Earth . 

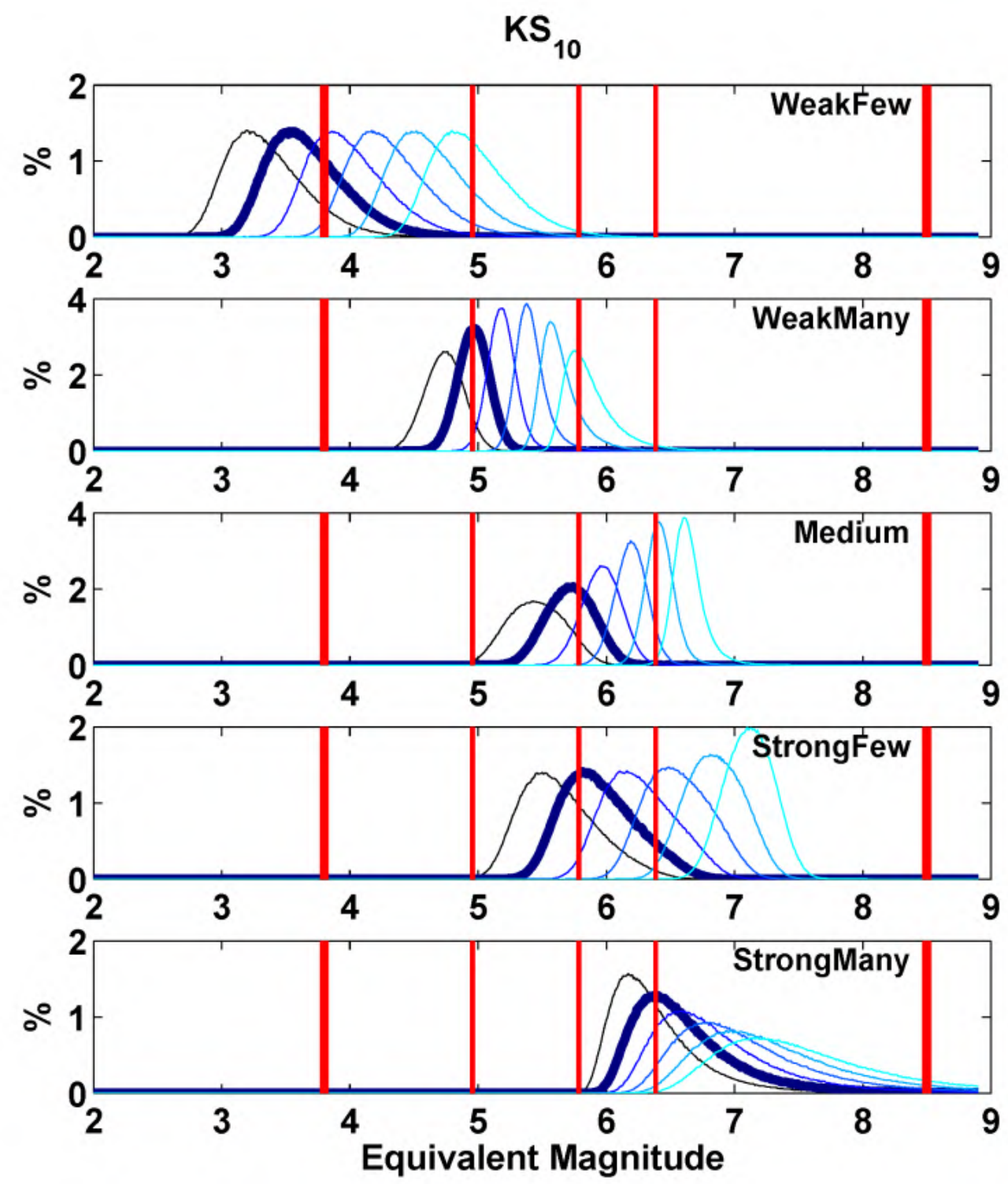
Seismic Moment Rate Estimation, v 2.0.3, last saved 08/01/2019 10:11:00
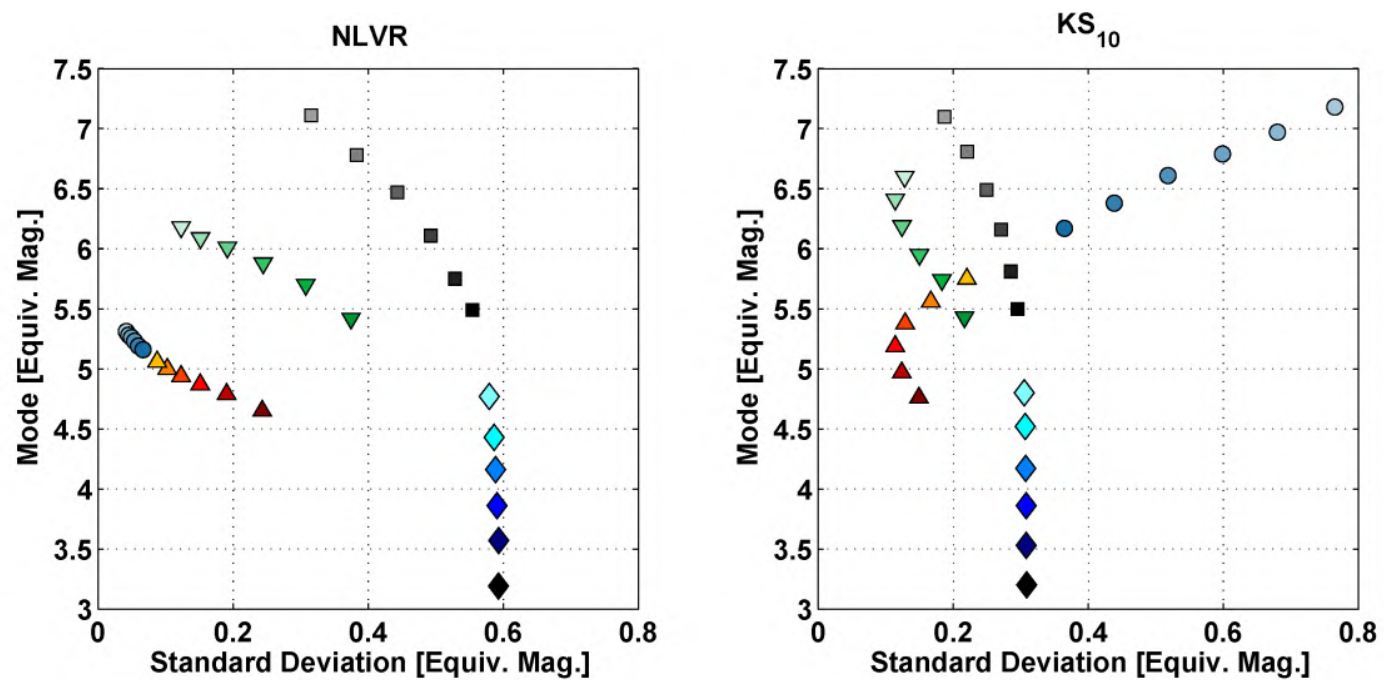

$1256 \quad$ Figure 15 Standard deviations and modes of the distribution densities shown in Figure 13 and Figure 14. Brigthter shades

1257 indicate longer registration times, symbols encode the end member models of Knapmeyer et al. (2006). Diamonds: WeakFew,

1258 Upward triangles: WeakMany Downward triangles: Medium, Circles: StrongMany, Squares: StrongFew

1259 
Seismic Moment Rate Estimation, v 2.0.3, last saved 08/01/2019 10:11:00

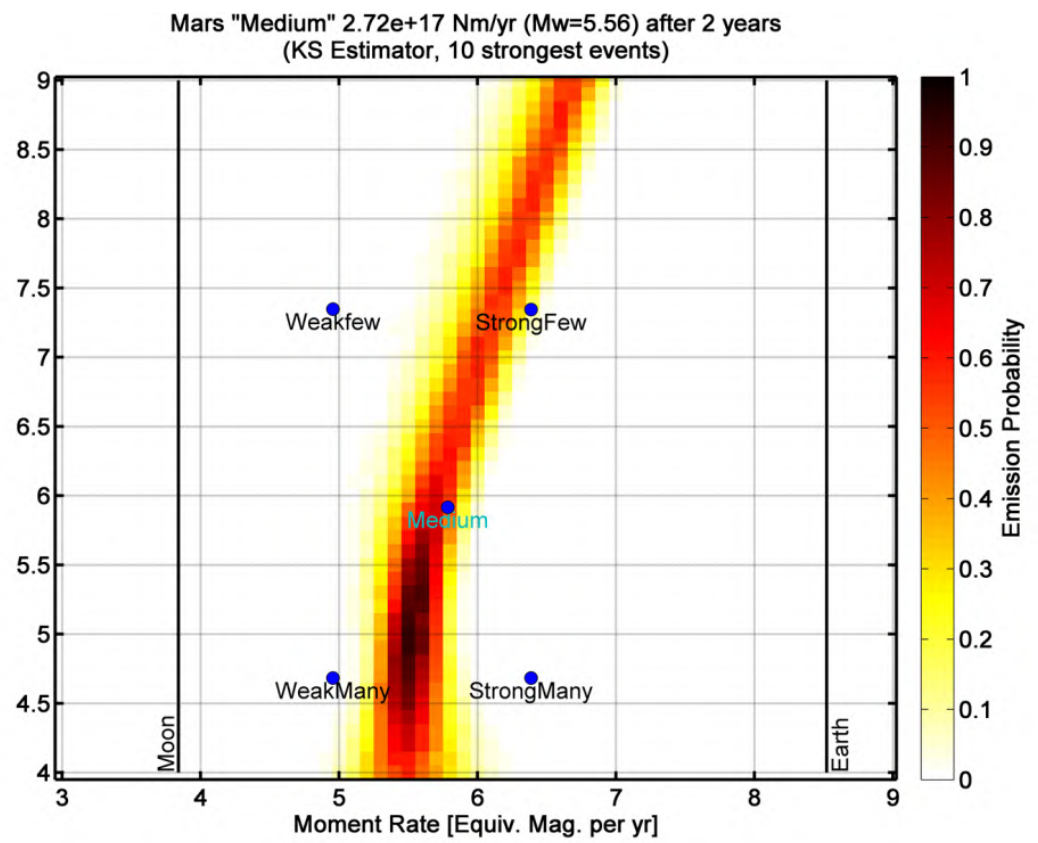

Figure $16 \mathrm{KS}_{10}$ emission probability for a synthetic Mars Medium catalog. Dots mark the moment rates and corner molisted in Table 1. 
Seismic Moment Rate Estimation, v 2.0.3, last saved 08/01/2019 10:11:00

1267

1268

page 75 of 76,16352 words 
Seismic Moment Rate Estimation, v 2.0.3, last saved 08/01/2019 10:11:00

1269

\section{Appendix}

1270 Flinn-Engdahl zones

1271 In the analysis of the GCMT catalog, we used the Flinn-Engdahl zonation scheme, in the revision of

1272 Young et al. (1995), to classify events as belonging to either subduction zones or mid-ocean ridges.

1273 We consider as mid-ocean ridge events all events in zones

$1274 \quad 143149150401401402403404405406408409410411413419424425426427428$

$1275429433634635636637638641648681682683688691692693694698699727 \quad 729735$

1276737738739753754

1277 We consider as subduction zone event all events in zones

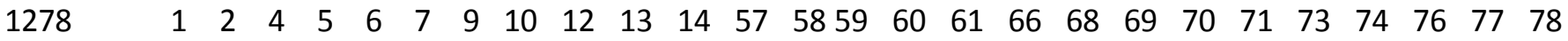

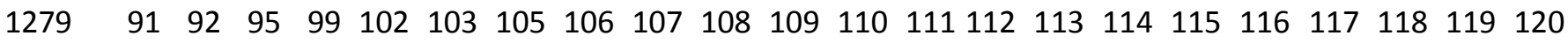

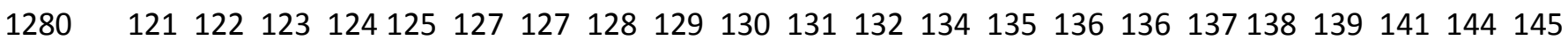
1281153159160169171171172173174175176176177178180181182183184185188189 1282191192193199200201202206209210211212214215216217218219220221223226 $1283 \quad 227228229231233234236237242244245247248249250255258261262263264265$ $1284 \quad 266268269271272273274275276277278279281282283284285286290366367368$ 1285387388396654657658659660685700701702703

1286 We consider as subducting slabs all events in the subduction zones as listed above, and deeper than $1287100 \mathrm{~km}$.

1288

page 76 of 76,16352 words 